\title{
Hypersonic Aerothermodynamics Analysis Across Nonequilibrium Regimes Using Continuum and Particle Methods
}

\author{
Andrew J. Lofthouse*, Leonardo C. Scalabrin ${ }^{\dagger}$ and Iain D. Boyd ${ }^{\ddagger}$ \\ Department of Aerospace Engineering, University of Michigan, Ann Arbor, 48109
}

\begin{abstract}
Hypersonic vehicles experience different flow regimes during flight due to changes in atmospheric density. Computational Fluid Dynamics (CFD) is not physically accurate in areas of highly non-equilibrium flows. The direct simulation Monte Carlo (DSMC) method, while physically accurate for all flow regimes, is relatively computationally expensive. In a continuing effort to understand the performance of CFD and DSMC in hypersonic flows, the current study investigates the effect of non-equilibrium on surface aerothermodynamic properties (pressure, shear stress and heat transfer rate) of a cylinder in Mach 10 and Mach 25 flows of nitrogen gas for several different flow regimes, from the continuum to a rarefied gas. Vibrational relaxation models used in each solution method are compared. CFD (with slip and temperature jump boundary conditions) and DSMC solutions are obtained at each condition. Non-equilibrium between internal energy modes and the translational mode do not have a large effect on surface property prediction, as total drag and peak heat transfer rate predictions by CFD remain within about $3 \%$ and $8 \%$, respectively, of the DSMC predictions for all regimes considered.
\end{abstract}

\section{Introduction}

The design of hypersonic vehicles requires accurate prediction of the surface properties. These quantities are typically the heat flux, pressure and shear stress. During it's trajectory through an atmosphere, a hypersonic vehicle will experience vastly different flow regimes due to the variation of atmospheric density with altitude. In addition, the high temperatures encountered due to the high velocities cause dissociation and ionization of the atmospheric gases. Reproduction of these varied flow conditions in ground-based laboratory facilities is both expensive and technically challenging. Hence, there is an extremely important role for computational models in the development of hypersonic vehicles.

In the continuum regime, characterized by very low Knudsen numbers, where the Knudsen number is defined in Eq. 1

$$
\mathrm{Kn}=\frac{\lambda}{L} \propto \frac{1}{\rho L}
$$

flows around hypersonic vehicles can be accurately simulated using traditional Computational Fluid Dynamics (CFD) by solving either the Euler or preferably the Navier-Stokes (NS) equations. The NS equations can be derived from kinetic theory based on the assumption of a small perturbation from an equilibrium velocity distribution function and linearly varying transport properties (viscosity, heat transfer coefficient and diffusion coefficient). ${ }^{1}$ Typical CFD methods also assume that the flow remains in thermodynamic equilibrium (the internal energy remains in equilibrium with the translational energy). In areas of the flow that include large gradients (such as the shock and boundary layers near the wall), these assumptions of equilibrium break down. In the rarefied flow regime (large Knudsen numbers) the flow can be computed using the direct

\footnotetext{
* Graduate Student, Senior Member AIAA.

${ }^{\dagger}$ Graduate Student, Member AIAA.

${ }^{\ddagger}$ Professor, Associate Fellow AIAA

"The views expressed in this paper are those of the author and do not reflect the official policy or position of the United States Air Force, Department of Defense, or the U.S. Government"
} 
simulation Monte Carlo (DSMC) method. ${ }^{2}$ The DSMC method does not depend assumptions involving a small perturbation from equilibrium and hence is more accurate than CFD methods for non-equilibrium flows. Generally speaking, CFD methods for solving the NS equations are about an order of magnitude faster than the DSMC method. Note that in continuum regimes, locally a flow may behave like a rarefied flow if the local characteristic length scale is very small. number. On a blunt body, a high-density fore-body flow can create a rarefied flow in the wake of the vehicle. In principle, the DSMC method can be applied to any dilute gas flow, but becomes prohibitively expensive for Knudsen numbers less than 0.001. Thus, it is attractive to find ways to increase the validity of CFD methods beyond the continuum regime.

It is important to be able to characterize the effect of a given level of non-equilibrium on the design variables of interest (here, these are all surface quantities: heat flux, pressure and shear stress). It has been shown that solutions of the Boltzmann equation are valid outside the continuum regime, whereas the Navier-Stokes equations are not. ${ }^{2}$ It has also been shown that solutions obtained with the DSMC method converge to solutions of the Boltzmann equation. ${ }^{3}$ Therefore, in this study we assume that the DSMC results are the more correct values. The objective, then, is to compare the results of DSMC and CFD and try to improve agreement even when encountering non-equilibrium.

The areas of the flow where the continuum hypothesis breaks down (or equivalently, where the flow is no longer in local thermodynamic equilibrium), can be quantified by the use of a continuum breakdown parameter. While there have been several breakdown parameters presented in the literature, ${ }^{4-7}$ the one that appears to be most appropriate for hypersonic compressible flows is the gradient-length local (GLL) Knudsen number ${ }^{8,9}$

$$
\mathrm{Kn}_{\mathrm{GLL}}=\frac{\lambda}{Q}\left|\frac{d Q}{d l}\right|
$$

where the derivative is taken in the direction of the maximum gradient, and $Q$ is some quantity of interest such as density, pressure, temperature or velocity magnitude. When calculating $\mathrm{Kn}_{\text {GLL }}$ based on velocity magnitude, the gradient is normalized by the maximum of the local velocity magnitude and the local speed of sound. It is generally assumed that continuum breakdown occurs whenever $\mathrm{Kn}_{\mathrm{GLL}}$ (based on the CFD solution) is greater than 0.05 .

Previous work ${ }^{10,11}$ sought to quantify the errors in CFD predictions of surface properties of a hypersonic blunt body by comparing those solutions with DSMC solutions for a variety of flows in the transitional regime. The maximum $\mathrm{Kn}_{\mathrm{GLL}}$ was also computed in each case to determine the effect of continuum breakdown on the surface properties. The cases considered were limited to a Mach 10 and Mach 25 flows of argon around a cylinder, with various slip boundary conditions imposed at the wall.

The current work builds on these results, with the main objective being to quantify any differences in agreement between the DSMC solutions and the CFD solutions when using nitrogen rather than argon as the working fluid. The use of a diatomic gas complicates the simulations due to the presence of rotational and vibrational energy modes in addition to the translational energy mode.

This paper will first briefly describe the simulation procedures used, including some background of the computational models. The vibrational relaxation models used here are then discussed and compared. We will then discuss general flow field and surface property results predicted by the models in several different flow regimes, from the continuum to a rarefied gas. Finally, some conclusions are presented.

\section{Background and Simulation Procedure}

This investigation considers a hypersonic flow of nitrogen over a two-dimensional, 12-inch diameter cylinder, as shown in Figure 1. Two different free stream velocities are used that correspond to Mach 10 and Mach 25 flows $\left(U_{\infty}=2883 \mathrm{~m} / \mathrm{s}\right.$ and $7208 \mathrm{~m} / \mathrm{s}$, respectively). The wall temperature is held constant at $500 \mathrm{~K}$ (Mach 10) and $1500 \mathrm{~K}$ (Mach 25). The free stream density of the flow is varied such that several different regimes are considered, from the continuum through the transitional to the rarefied regime, as shown in Table 1. Knudsen numbers are calculated based on freestream conditions and the cylinder diameter, using the hard-sphere model for the mean free path calculation. Surface and flow field properties for this flow are presented from two different computational approaches.

First, DSMC results are provided from the MONACO code $^{12}$ for the flow conditions mentioned above. MONACO is a general 2D/3D, object-oriented, cell-based, parallel implementation of the DSMC method. It uses the Variable Hard Sphere (VHS) and Variable Soft Sphere collision models. ${ }^{2,13}$ It also includes variable vibrational ${ }^{14}$ and rotational ${ }^{15}$ energy exchange probability models. 


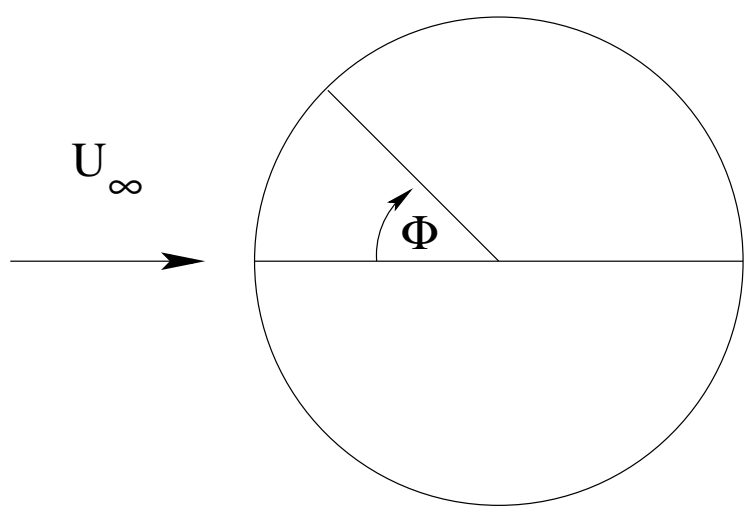

Figure 1. Geometry definition.

All MONACO solutions are generated using a fixed wall temperature at $500 \mathrm{~K}$ and $1500 \mathrm{~K}$. Bird's variable hard sphere model is used. ${ }^{2}$ The VHS parameters used in the DSMC computations are those for standard nitrogen (the temperature exponent, $\omega$, is 0.7 with a reference diameter of $4.11 \times 10^{-10} \mathrm{~m}$ at a reference temperature of $290 \mathrm{~K}$ ). In general, the mesh used for the final solution for each case is adapted from previous solutions such that each cell size is on the order of a mean free path. The exceptions are the $\mathrm{Kn}=0.002$ cases, where the cell size is approximately six times the mean free path, and the $\mathrm{Kn}=0.01$ cases, where the cell sizes near the surface are on the order of two mean free paths in size. In all cases the subcell method is used to select particles for collisions ${ }^{2}$ to ensure physical accuracy.

Second, CFD results are obtained through solution of the Navier-Stokes equations. The CFD results are obtained using the Michigan Aerothermodynamic Navier-Stokes (LeMANS) code, developed at the University of Michigan for the simulation of hypersonic reacting flow-fields. ${ }^{16,17}$ LeMANS is a general two-dimensional, three-dimensional and axisymmetric, parallel, unstructured, finite-volume CFD code capable of simulating gases in thermal and chemical non-equilibrium. A two-temperature model is used to account for the nonequilibrium between the vibrational and the translational-rotational modes, ${ }^{18}$ with the energy exchange rates modeled using the Landau-Teller model. ${ }^{1}$ Standard finite-rate chemistry models for reacting air using 5 species, 7 species and 11 species allow atmospheric flow simulations in conditions causing dissociation and weak ionization. Although transport properties can be computed using Wilke's ${ }^{19}$ and Blottner's ${ }^{20}$ models, it is important to ensure identical transport properties are used with both models, hence the VHS model viscosity is used directly in the CFD calculations. ${ }^{21}$ The thermal conductivity is determined using Eucken's relation. ${ }^{16}$

All LeMANS solutions are generated assuming an isothermal wall at $500 \mathrm{~K}$ (Mach 10) and $1500 \mathrm{~K}$ (Mach 25). Different boundary conditions, including no-slip and slip velocity and temperature jump are enforced. These boundary conditions are discussed in greater detail in the previous work. ${ }^{10}$ In the results that follow, Type 0 refers to the no-slip condition; Type 2 refers to the Gökçen-type slip conditions; ${ }^{22}$ and Type 3 refers to the Knudsen-layer correction to the standard slip conditions proposed by Lockerby et al. ${ }^{23}$ Solutions computed using the Type 1, or simple Maxwell-type slip conditions, exhibited large fluctuations in temperature in several cases using $\mathrm{N}_{2}$ and these conditions are not considered further here.

The addition of a vibrational temperature in the CFD method requires an additional temperature jump relation. For the cases considered here, the vibrational temperature jump is computed using similar relations to those used for the translational/rotational temperature jump. ${ }^{24}$ 


\section{A. Vibrational Relaxation}

The rate of energy exchange between the translational and vibrational modes is inversely proportional to the vibrational relaxation time, $\tau_{v}$. The Landau-Teller model is generally used to approximate the vibrational relaxation time. ${ }^{1}$ Millikan and White ${ }^{25}$ correlated experimental data with the Landau-Teller model to obtain an expression for the vibrational relaxation time as

$$
\tau_{L T}=\frac{1}{p} \exp \left(\frac{A}{T^{1 / 3}}+B\right)
$$

where $p$ is the pressure, $T$ is the temperature and $A$ and $B$ are constants that vary by species. The values for $A$ and $B$ for $\mathrm{N}_{2}$ are 220 and -24.80 , respectively, for a pressure given in atm. For a pressure given in $\mathrm{Pa}$, the value for $B$ is -13.27 .

The Park correction, ${ }^{18}$ which is necessary for temperatures typically encountered at hypersonic speeds, is given as

$$
\tau_{P}=\frac{1}{n C \sigma}
$$

where $n$ is the particle density, $C$ is the mean molecular speed and $\sigma$ is the vibrational collision cross section. The current work uses a constant vibrational collision cross section, $\sigma=5.81 \times 10^{-21}$, for both the CFD and DSMC simulations.

The vibrational relaxation time is then given in Eq. 5.

$$
\tau_{v}=\tau_{L T}+\tau_{P}
$$

The Landau-Teller model is a continuum model and can be directly implemented in a CFD code. LeMANS uses the Millikan and White values in the Landau-Teller mode, although with a different definition of $B$ that corresponds directly to $B=-24.8$, as given above.

The vibrational relaxation process in DSMC is governed by collision probabilities. The probability that a collision will result in an exchange of vibrational energy is given as

$$
P=\frac{1}{\tau_{v} \nu}
$$

where $\nu$ is the bi-molecular collision rate. This probability is then implemented in MONACO using discrete collision probabilities ${ }^{14,26}$ (with various corrections ${ }^{27,28}$ ), which, when integrated over all collisions, is expected to correspond to the total probability as given in Eq. 6 .

A recent study compared the theoretical vibrational probability (Eq. 6) with that given by MONACO. ${ }^{29}$ This study showed that, while remaining within a factor of 2 , the overall probability seen in MONACO differed from the theoretical probability as shown in Table 2. The discrepancies between MONACO and the theoretical vibrational probability is most likely due to the method of analytical integration used in deriving the discrete collision probabilities used in the DSMC model. ${ }^{26}$ In the present investigation, the vibrational collision probability is multiplied by a factor (as shown in the fourth col-

Table 2. Vibrational collision probability in MONACO compared to theory for $\mathrm{N}_{2}-\mathrm{N}_{2}$ collisions. ${ }^{29}$

\begin{tabular}{|c|c|c|c|}
\hline Temperature $(\mathrm{K})$ & MONACO & Theory & Factor \\
\hline 5000 & 0.0002 & 0.0001 & 0.5 \\
10000 & 0.0017 & 0.0024 & 1.41 \\
15000 & 0.0042 & 0.0077 & 1.83 \\
20000 & 0.0068 & 0.0123 & 1.81 \\
25000 & 0.0090 & 0.0153 & 1.7 \\
\hline
\end{tabular}
umn of Table 2) to better correspond to theory. The factor is chosen based on the maximum translational temperature in each case; for the Mach 10 cases the maximum temperature is approximately $5,000 \mathrm{~K}$, so the vibrational collision probability is multiplied by 0.5 , and for the Mach 25 cases the maximum temperature is approximately 25,000 and the vibrational collision probability is multiplied by 1.7 .

\section{Results}

This investigation seeks to quantify the differences in the surface properties of a cylinder in a hypersonic flow by comparing CFD and DSMC predictions for total drag and peak heat transfer. Additionally, the 
overall flow features, such as the temperature field, are compared, as well as the surface distributions of pressure, shear stress and heat transfer.

Tables 3 and 4 compare the total drag and the peak heat transfer rate predicted by both computational methods. Here, the peak heat transfer rate is obtained by averaging over the surface area within one degree of the stagnation point. For CFD, these quantities are calculated for each of the different boundary conditions implemented (with the exception of the Type 1 condition, as mentioned previously).

Although here a gas with internal degrees of freedom is considered, the additional possibility of thermodynamic non-equilibrium does not seem to significantly affect the surface properties, or the total drag and stagnation heat transfer. The total drag and stagnation heat transfer results from the previous investigation that considered argon are reproduced here in Tables 5 and 6 . As with the previous work, the slip boundary conditions improve the agreement between the two simulation methods, with the Type 2 slip boundary conditions showing the best agreement. In some cases, there is better agreement with the $\mathrm{N}_{2}$ cases than with the Ar cases; for example, for the Mach 10, $\mathrm{Kn}=0.25$ case, the total drag computed using the Type 2 boundary conditions is within $3 \%$ of the DSMC solution with $\mathrm{N}_{2}$ although it is just over $5 \%$ when using argon.

The peak heat transfer rates also shows similar trends with $\mathrm{N}_{2}$ as with Ar, although here the largest difference seen in the $\mathrm{N}_{2}$ results using the Type 2 boundary conditions is $8 \%$, compared to about $6 \%$ with Ar. The Mach 25 heat transfer results show a larger difference when considering $\mathrm{N}_{2}$, although the differences are still within $3 \%$. These larger differences are most likely due to computational artifacts, rather than complexity due to the non-equilibrium nature of the flow, as will be discussed below.

Overall, however, the current results for $\mathrm{N}_{2}$ are very similar to those for Ar. The Type 2 conditions give the best results, with total drag being within $3 \%$ of the DSMC results, and the peak heating rates being within about $5 \%$.

Given that the Type 2 condition shows the best agreement with the DSMC solutions, the CFD field results shown below are taken from the Type 2 CFD case.

Table 3. Total drag (using $\mathrm{N}_{2}$ as working fluid; current work).

Mach 10

\begin{tabular}{|c|c|c|c|c|c|}
\hline \multirow{2}{*}{$\mathrm{Kn}_{\infty}$} & \multicolumn{5}{|c|}{ Drag/Length [N/m] (\% Difference) } \\
\cline { 2 - 6 } & DSMC & CFD (0) & CFD (1) & CFD (2) & CFD (3) \\
\hline 0.002 & 162.4 & $162.4(0.0 \%)$ & N/A & $162.2(-0.2 \%)$ & $162.4(0.0 \%)$ \\
0.01 & 34.23 & $34.37(0.4 \%)$ & N/A & $34.17(-0.2 \%)$ & $34.32(0.3 \%)$ \\
0.05 & 7.346 & $7.780(5.9 \%)$ & N/A & $7.456(1.5 \%)$ & $7.603(3.5 \%)$ \\
0.25 & 1.714 & $2.056(19.9 \%)$ & N/A & $1.702(-0.7 \%)$ & $1.868(9.0 \%)$ \\
\hline
\end{tabular}

Mach 25

\begin{tabular}{|c|c|c|c|c|c|}
\hline \multirow{2}{*}{$\mathrm{Kn}_{\infty}$} & \multicolumn{5}{|c|}{ Drag/Length [N/m] (\% Difference) } \\
\cline { 2 - 6 } & DSMC & CFD (0) & CFD (1) & CFD (2) & CFD (3) \\
\hline 0.002 & 1,020 & $1,022(0.2 \%)$ & N/A & $1,021(0.1 \%)$ & $1,022(0.2 \%)$ \\
0.01 & 210.1 & $213.6(1.7 \%)$ & N/A & $212.7(1.2 \%)$ & $213.4(1.6 \%)$ \\
0.05 & 46.36 & $49.00(5.7 \%)$ & N/A & $47.07(1.5 \%)$ & $48.02(3.6 \%)$ \\
0.25 & 10.94 & $13.5(23.4 \%)$ & N/A & $11.25(2.8 \%)$ & $12.21(11.6 \%)$ \\
\hline
\end{tabular}

The breakdown parameter is calculated using both the CFD and the DSMC solutions using Equation 2 with the derivative being taken in the direction of the steepest gradient. As with previous work, the only causes of breakdown to the continuum hypothesis expected here are in regions of high gradients (such as the shock and boundary layer) and regions of rarefaction (such as in the wake). Thermal non-equilibrium is expected to be higher in regions where the breakdown parameter values are highest; thus, the non-equilibrium between translational and rotational modes is expected in the shock wave, and the wake. As the flow becomes more rarefied in the higher Knudsen-number cases, the thermal non-equilibrium is expected to increase. 
Table 4. Peak heat transfer rate (using $\mathrm{N}_{2}$ as working fluid; current work).

\section{Mach 10}

\begin{tabular}{|c|c|c|c|c|c|}
\hline \multirow{2}{*}{$\mathrm{Kn}_{\infty}$} & \multicolumn{5}{|c|}{ Peak Heating $\left[\mathrm{kW} / \mathrm{m}^{2}\right]$ (\% Difference) } \\
\cline { 2 - 6 } & DSMC & CFD (0) & CFD (1) & CFD (2) & CFD (3) \\
\hline 0.002 & 69.88 & $69.14(-1.1 \%)$ & N/A & $68.74(-1.6 \%)$ & $68.62(-1.8 \%)$ \\
0.01 & 31.20 & $31.01(-0.6 \%)$ & N/A & $30.55(-2.1 \%)$ & $30.55(-2.1 \%)$ \\
0.05 & 13.28 & $14.50(9.2 \%)$ & N/A & $13.85(4.3 \%)$ & $14.09(6.1 \%)$ \\
0.25 & 5.173 & $6.380(23.3 \%)$ & N/A & $5.508(6.5 \%)$ & $6.034(16.6 \%)$ \\
\hline
\end{tabular}

\section{Mach 25}

\begin{tabular}{|c|c|c|c|c|c|}
\hline \multirow{2}{*}{$\mathrm{Kn}_{\infty}$} & \multicolumn{5}{|c|}{ Peak Heating $\left[\mathrm{kW} / \mathrm{m}^{2}\right](\%$ Difference) } \\
\cline { 2 - 6 } & DSMC & CFD $(0)$ & CFD $(1)$ & CFD $(2)$ & CFD $(3)$ \\
\hline 0.002 & 1,348 & $1,309(-2.9 \%)$ & N/A & $1,303(-3.3 \%)$ & $1,302(-3.4 \%)$ \\
0.01 & 585.0 & $586.3(0.2 \%)$ & N/A & $579.7(-0.9 \%)$ & $579.4(-1.0 \%)$ \\
0.05 & 258.1 & $281.7(9.1 \%)$ & N/A & $268.6(4.1 \%)$ & $274.6(6.4 \%)$ \\
0.25 & 91.87 & $118.3(28.8 \%)$ & N/A & $99.10(7.9 \%)$ & $111.6(21.5 \%)$ \\
\hline
\end{tabular}

Table 5. Total drag (using Ar as working fluid; reproduced from previous work ${ }^{10}$ ).

\section{Mach 10}

\begin{tabular}{|c|c|c|c|c|c|}
\hline \multirow{2}{*}{$\mathrm{Kn}_{\infty}$} & \multicolumn{5}{|c|}{ Drag/Length $[\mathrm{N} / \mathrm{m}](\%$ Difference) } \\
\cline { 2 - 6 } & DSMC & CFD (0) & CFD (1) & CFD (2) & CFD (3) \\
\hline 0.002 & 187.6 & $187.5(-0.1 \%)$ & $187.4(-0.1 \%)$ & $187.4(-0.1 \%)$ & $187.6(0.0 \%)$ \\
0.01 & 40.02 & $40.32(0.8 \%)$ & $40.19(0.4 \%)$ & $40.16(0.4 \%)$ & $40.27(0.6 \%)$ \\
0.05 & 8.900 & $9.416(5.8 \%)$ & $9.122(2.5 \%)$ & $8.863(-0.4 \%)$ & $9.078(2.0 \%)$ \\
0.25 & 2.092 & $2.585(23.6 \%)$ & $2.301(10.0 \%)$ & $1.982(-5.2 \%)$ & $2.253(7.7 \%)$ \\
\hline
\end{tabular}

\section{Mach 25}

\begin{tabular}{|c|c|c|c|c|c|}
\hline \multirow{2}{*}{$\mathrm{Kn}_{\infty}$} & \multicolumn{5}{|c|}{ Drag/Length [N/m] (\% Difference) } \\
\cline { 2 - 6 } & DSMC & CFD (0) & CFD (1) & CFD (2) & CFD (3) \\
\hline 0.002 & 1,171 & $1,176(0.4 \%)$ & $1,176(0.4 \%)$ & $1,176(0.4 \%)$ & $1,177(0.5 \%)$ \\
0.01 & 250.8 & $255.3(1.8 \%)$ & $254.6(1.5 \%)$ & $254.4(1.4 \%)$ & $255.1(1.7 \%)$ \\
0.05 & 56.85 & $61.17(7.6 \%)$ & $59.38(4.5 \%)$ & $57.04(0.3 \%)$ & $58.90(3.6 \%)$ \\
0.25 & 13.35 & $17.61(32.0 \%)$ & $15.73(17.8 \%)$ & $13.02(-2.4 \%)$ & $15.14(13.4 \%)$ \\
\hline
\end{tabular}


Table 6. Peak heat transfer rate (using Ar as working fluid; reproduced from previous work ${ }^{10}$ ).

Mach 10

\begin{tabular}{|c|c|c|c|c|c|}
\hline \multirow{2}{*}{$\mathrm{Kn}_{\infty}$} & \multicolumn{5}{|c|}{ Peak Heating $\left[\mathrm{kW} / \mathrm{m}^{2}\right]$ (\% Difference $)$} \\
\hline & DSMC & CFD (0) & CFD (1) & CFD (2) & CFD (3) \\
\hline 0.002 & 89.80 & $89.85(0.0 \%)$ & $89.14(-0.7 \%)$ & $89.00(-0.9 \%)$ & $88.54(-1.4 \%)$ \\
\hline 0.01 & 39.13 & $40.22(2.8 \%)$ & $39.49(0.9 \%)$ & $39.20(0.2 \%)$ & $38.89(-0.6 \%)$ \\
\hline 0.05 & 15.92 & $18.08(13.6 \%)$ & $17.25(8.4 \%)$ & $15.79(-0.8 \%)$ & $16.81(5.6 \%)$ \\
\hline 0.25 & 5.926 & $7.851(32.5 \%)$ & $7.061(19.1 \%)$ & $6.184(4.3 \%)$ & $6.640(12.1 \%)$ \\
\hline
\end{tabular}

Mach 25

\begin{tabular}{|c|c|c|c|c|c|}
\hline \multirow{2}{*}{$\mathrm{Kn}_{\infty}$} & \multicolumn{5}{|c|}{ Peak Heating $\left[\mathrm{kW} / \mathrm{m}^{2}\right]$} \\
\cline { 2 - 6 } & DSMC & CFD (0) & CFD (1) & CFD (2) & CFD (3) \\
\hline 0.002 & 1,746 & $1,763(0.9 \%)$ & $1,750(0.2 \%)$ & $1,746(0.0 \%)$ & $1,739(-0.4 \%)$ \\
0.01 & 749.6 & $791.0(5.5 \%)$ & $777.4(3.7 \%)$ & $768.4(2.5 \%)$ & $765.3(2.1 \%)$ \\
0.05 & 309.9 & $357.9(13.6 \%)$ & $341.0(10.4 \%)$ & $294.2(-4.8 \%)$ & $331.2(7.2 \%)$ \\
0.25 & 105.7 & $147.8(39.9 \%)$ & $133.6(19.1 \%)$ & $111.7(5.7 \%)$ & $123.7(17.0 \%)$ \\
\hline
\end{tabular}

In the results that follow, the surface properties are presented in terms of non-dimensional coefficients for surface pressure, shear stress and heat transfer with the following definitions:

$$
\begin{aligned}
C_{P} & =\frac{p-p_{\infty}}{\frac{1}{2} \rho_{\infty} U_{\infty}^{2}} \\
C_{F} & =\frac{\tau}{\frac{1}{2} \rho_{\infty} U_{\infty}^{2}} \\
C_{H} & =\frac{q}{\frac{1}{2} \rho_{\infty} U_{\infty}^{3}}
\end{aligned}
$$

where $p$ is the pressure, $\tau$ is the shear stress, $q$ is the heat transfer rate, $p_{\infty}$ is the free stream pressure, $\rho_{\infty}$ is the free stream density and $U_{\infty}$ is the free stream velocity. The surface properties in each case are plotted as a function of the angle around the cylinder, with the stagnation point being located at an angle of zero (see Figure 1).

Along with the surface properties, the maximum $\mathrm{Kn}_{\mathrm{GLL}}$ at the surface (based on the CFD solution with no-slip condition) is also plotted in each case.

All temperature results from the DSMC simulations are obtained by averaging the translational and rotational temperatures, as shown in Eq. 10 unless otherwise noted.

$$
T=\frac{3 T_{t r a}+2 T_{r o t}}{5}
$$

\section{IV. $\quad \mathrm{Kn}=0.002$}

The simulations for the Knudsen number of 0.002 cases show very similar trends as shown in the previous work. ${ }^{10}$ Although the flow is within the continuum, no-slip regime, there is evidence of continuum breakdown in the shock, along the cylinder surface in the boundary layer and in the wake as seen in Figure 2. There does not seem to be a larger amount of non-equilibrium predicted by the breakdown parameter for $\mathrm{N}_{2}$ than there was using Ar.

The different temperatures along the stagnation line (including the translational, rotational and vibrational temperatures for the DSMC solutions; and the translational/rotational and vibrational temperatures for the CFD solutions) are compared in Figure 3. There is a very small amount of thermal non-equilibrium 
CFD

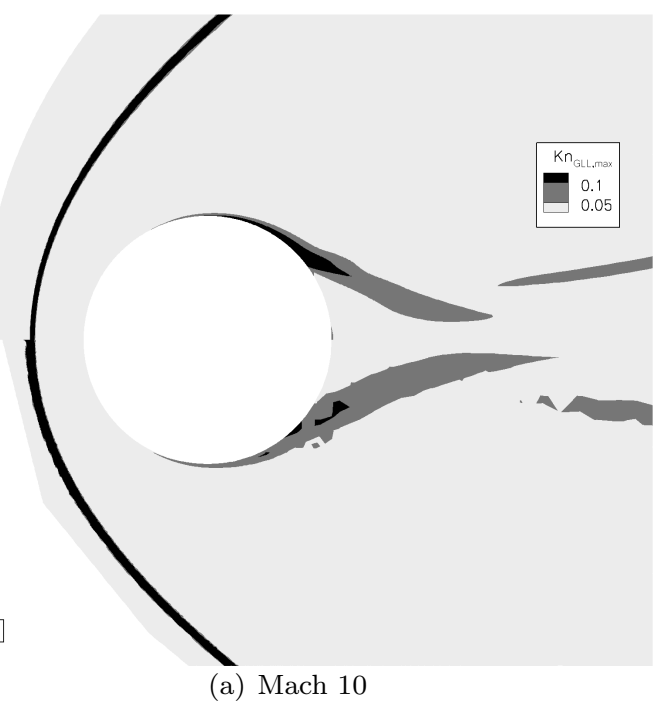

CFD

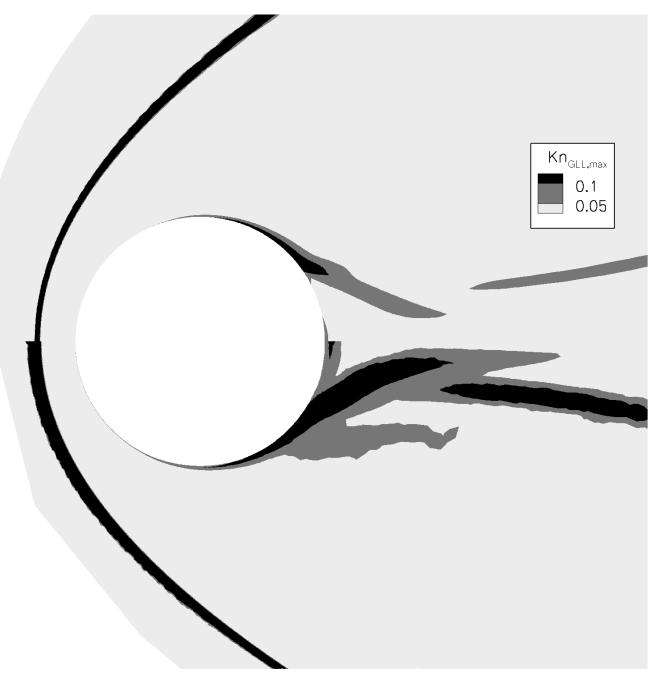

(b) Mach 25

Figure 2. $\mathrm{Kn}_{\infty}=\mathbf{0 . 0 0 2} \mathrm{Kn}_{\mathrm{GLL}}$ for Mach 10 and Mach 25. Light gray regions correspond to $\mathrm{Kn}_{\mathrm{GLL}}<0.05$, dark gray regions correspond to $0.05<\mathrm{Kn}_{\mathrm{GLL}}<0.10$ and black regions correspond to $\mathrm{Kn}_{\mathrm{GLL}}>0.10$.

between the translational and rotational modes for the Mach 10 case, with a larger amount of non-equilibrium between those two modes for the Mach 25 case. Nevertheless, there is very good agreement between CFD and DSMC for the average translational/rotational temperatures and for the vibrational temperatures.

The surface pressure distributions (not shown) predicted by both methods agree very well, for all flows and boundary conditions considered. The surface shear stress, shown in Figure 4, also shows good agreement between the two methods, with some slight differences between $\Phi=45$ and 90 degrees. However, the heat transfer rates seen in Figure 5 show larger differences than would be expected at this relatively high density flow. CFD predictions of heat transfer rate are very sensitive to grid alignment and numerical dissipation in the numerical algorithm. Additionally, DSMC predictions of heat transfer rate are sensitive to the cell sizes and the number of particles per cell. Although there are sufficient numbers of particles per cell in the present simulations, the cell sizes of approximately 6 mean free paths might be too large to accurately predict the heat transfer rate in the stagnation region. Further simulations, with suitably refined meshes in the case of DSMC and suitable values of numerical dissipation in the case of CFD, are expected to improve the agreement to within about $1 \%$, although the current differences of about $3 \%$ are reasonable. Nevertheless, the total drag agreement is excellent, with less than $1 \%$ difference.

\section{V. $\mathrm{Kn}=0.01$}

For a flow with Knudsen number of 0.01, there is increased evidence of non-equilibrium, as shown in Figure 6, which is very similar to that obtained previously. Comparison of general flow field features shows that the two numerical solutions (CFD and DSMC) do not differ much. The stagnation line temperatures are shown Figure 7, with the translational/rotational temperature fields shown in Figure 8 and the vibrational temperature fields shown in Figure 9. The more rarefied conditions lead to a thicker shock, with larger amounts of thermal non-equilibrium. There is more excitation of the vibrational mode in the Mach 25 flow, although the more rarefied conditions, with fewer collisions, lead to less vibrational excitation than seen in the $\mathrm{Kn}=0.002$ case. However, there is still excellent agreement between the two computational methods, with some small differences in temperature behind the shock for the Mach 25 case. The vibrational temperature fields also differ by a small amount in the wake for the Mach 10 case. This is most likely due to the temperature jump condition being imposed at the wall.

Figure 9(a) apparently shows a discrepancy in the free stream vibrational temperatures used in the Mach 10 CFD and DSMC simulations. This is due to the inherent differences in the continuum and particle computational methods. In the CFD method, the vibrational temperature is explicitly set equal to the translational/rotational temperature, while the DSMC method assumes that the vibrational modes of the free stream particles are initially in an un-excited state. As the particles undergo collisions, the vibrational 


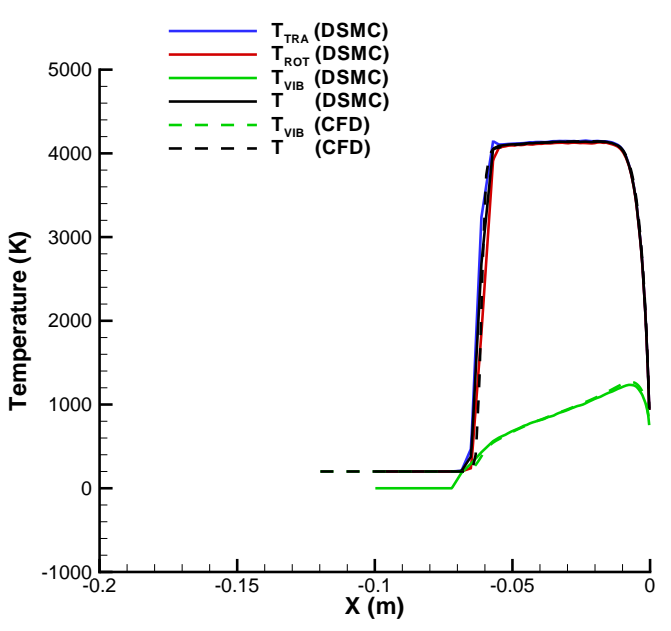

(a) Mach 10

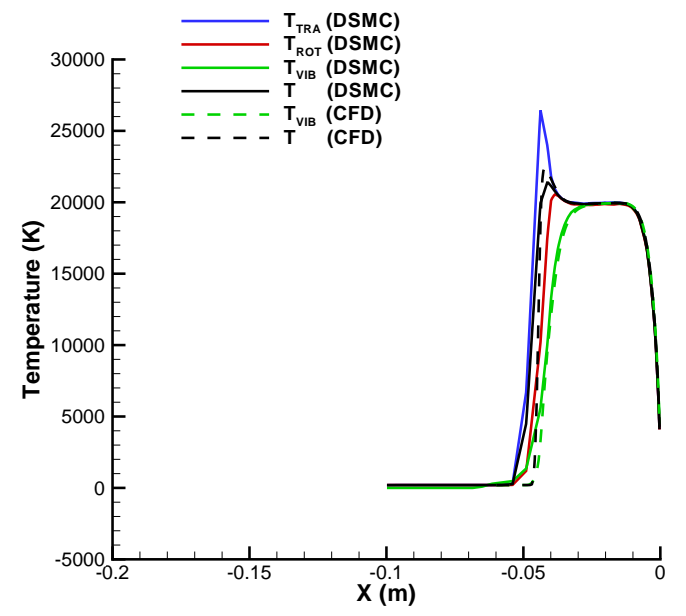

(b) Mach 25

Figure 3. $\mathrm{Kn}_{\infty}=0.002$ temperatures along stagnation streamline, for Mach 10 and Mach 25.

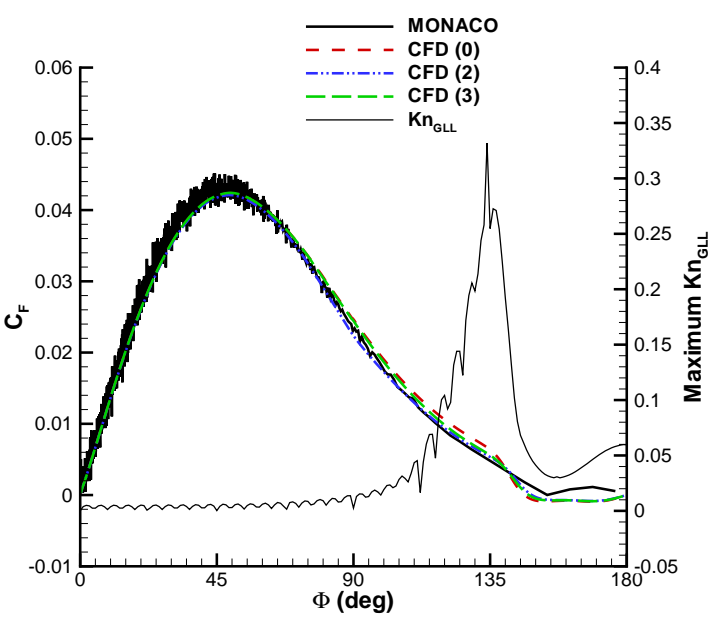

(a) Mach 10

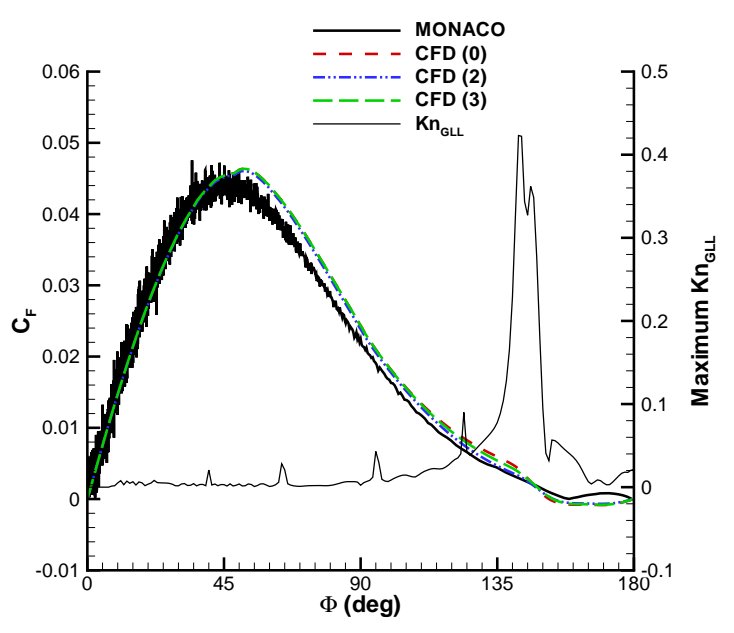

(b) Mach 25

Figure 4. $\mathrm{Kn} \mathrm{n}_{\infty}=\mathbf{0 . 0 0 2}$ surface friction coefficient and $\mathrm{Kn} \mathrm{GLL}_{\mathrm{GL}}$ for Mach 10 and Mach 25. 


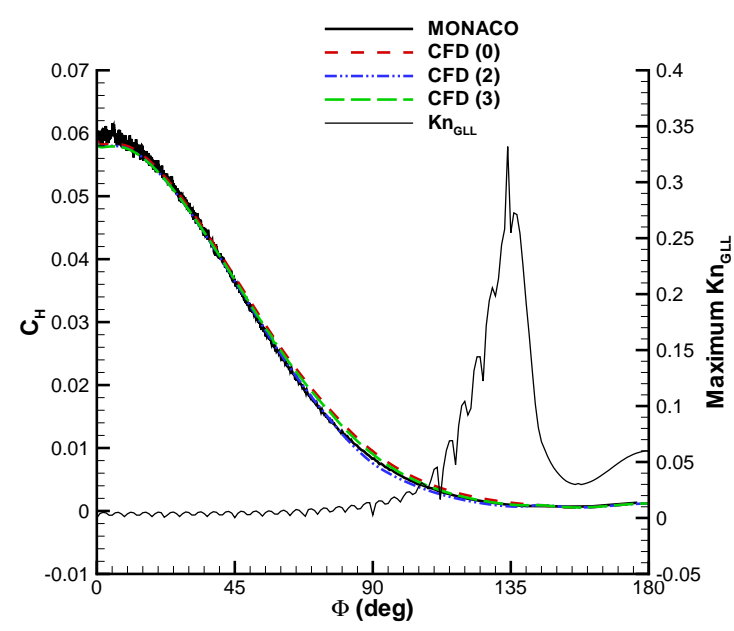

(a) Mach 10

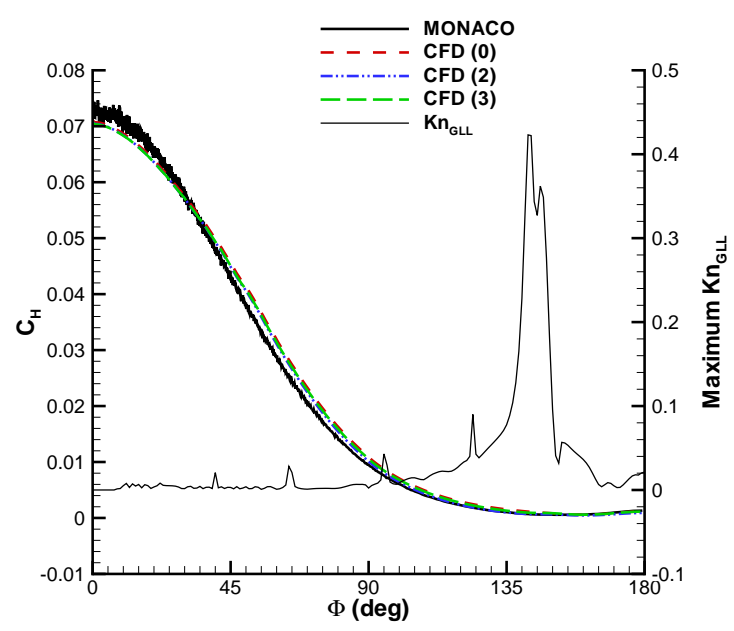

(b) Mach 25

Figure 5. $K \mathrm{n}_{\infty}=0.002$ surface heating coefficient and $\mathrm{Kn}_{\mathrm{GLL}}$ for Mach 10 and Mach 25.

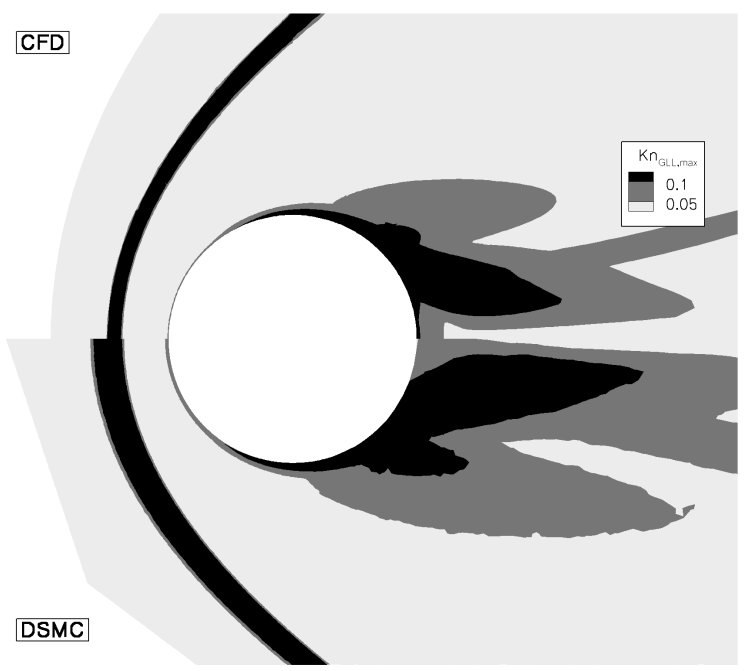

(a) Mach 10

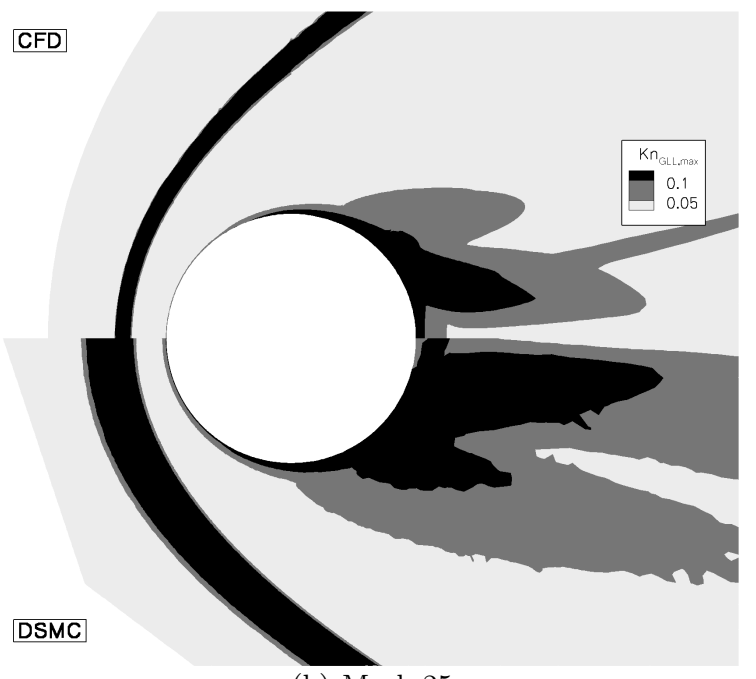

(b) Mach 25

Figure 6. $\mathrm{Kn}_{\infty}=0.01 \mathrm{Kn}_{\mathrm{GLL}}$ for Mach 10 and Mach 25. Light gray regions correspond to $\mathrm{Kn}_{\mathrm{GLL}}<0.05$, dark gray regions correspond to $0.05<\mathrm{Kn}_{\mathrm{GLL}}<0.10$ and black regions correspond to $\mathrm{Kn}_{\mathrm{GLL}}>0.10$. 
energy, and hence the vibrational temperature, relaxes to an appropriate value for current translational and rotational temperatures. For the case of a Mach 10 flow, the free stream temperature is low enough that there are very few, if any, particles whose vibrational modes are excited. This remains so until the particles encounter the higher temperatures within the shock, at which point there are a significant number of particles with excited vibrational modes. The streaks in the vibrational temperature field are the locations where there are some particles with excited vibrational modes. This also explains the slight dip in the DSMC vibrational temperature along the stagnation line shown in Figure 7.

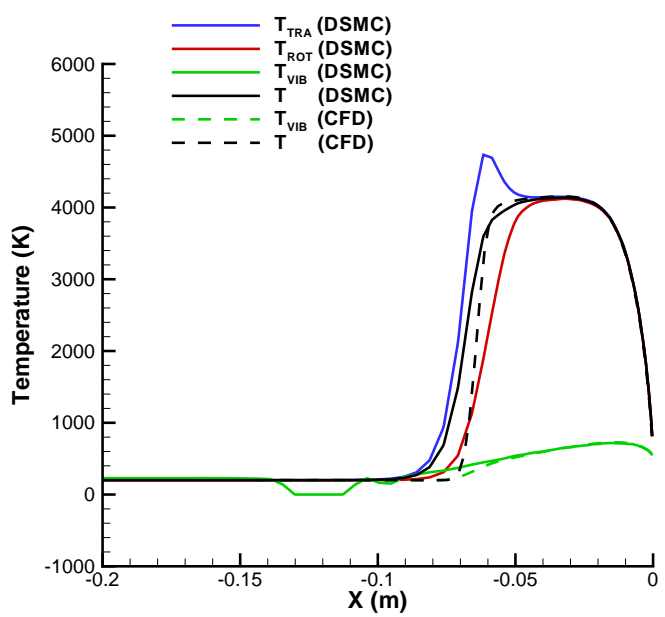

(a) Mach 10

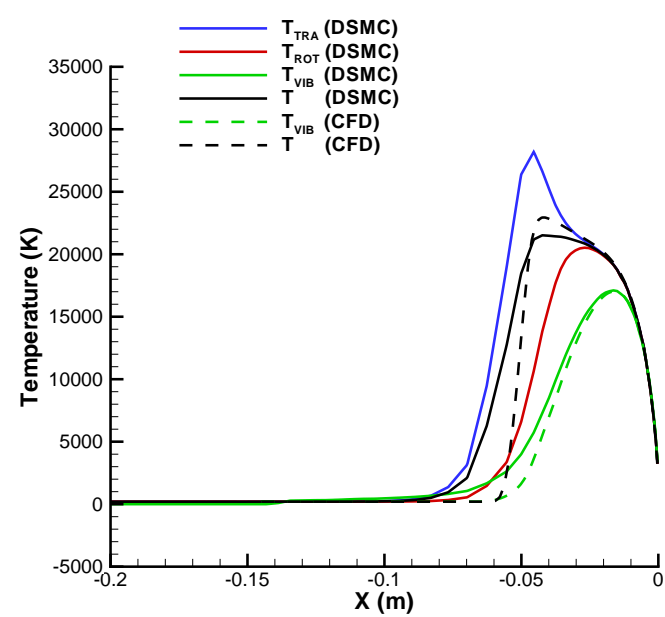

(b) Mach 25

Figure 7. $\mathrm{Kn}_{\infty}=0.01$ temperatures along stagnation streamline, for Mach 10 and Mach 25.

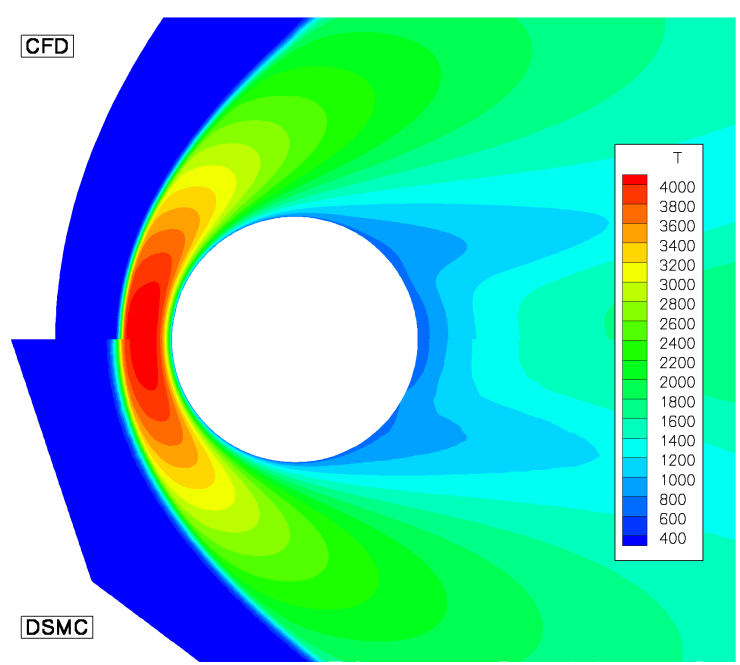

(a) Mach 10

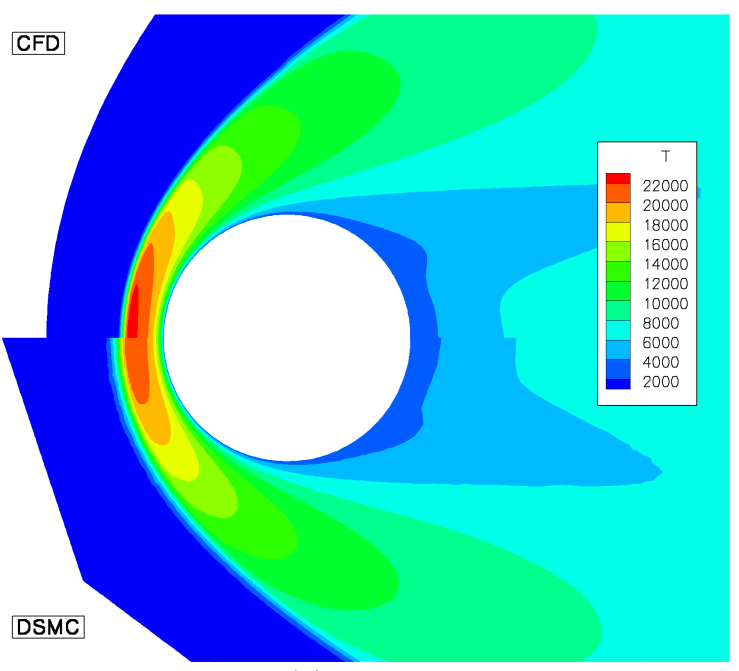

(b) Mach 25

Figure 8. $\mathrm{Kn}_{\infty}=0.01$ rotational/translational temperature field for Mach 10 and Mach 25.

The amount of non-equilibrium in the shock and near the surface does not seem to significantly affect the surface properties, shown in Figures 10 - 11 (the surface pressure distribution is not shown here). The total drag predicted by CFD is still within $1 \%$ of that predicted by DSMC, for Mach 10 , and within $2 \%$ for Mach 25, as shown in Table 3 . The peak heating also differs by about $2 \%$ for all cases. 


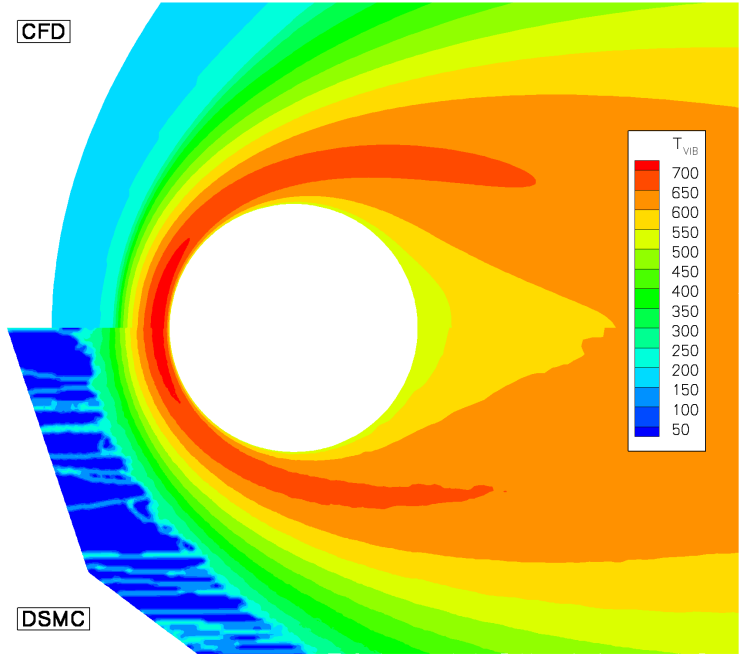

(a) Mach 10

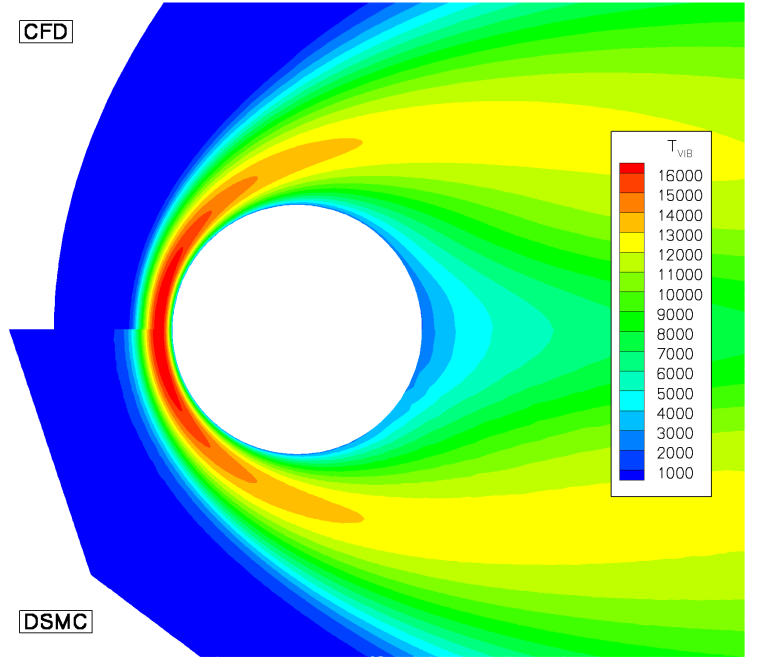

(b) Mach 25

Figure 9. $K \mathrm{n}_{\infty}=0.01$ vibrational temperature field for Mach 10 and Mach 25.

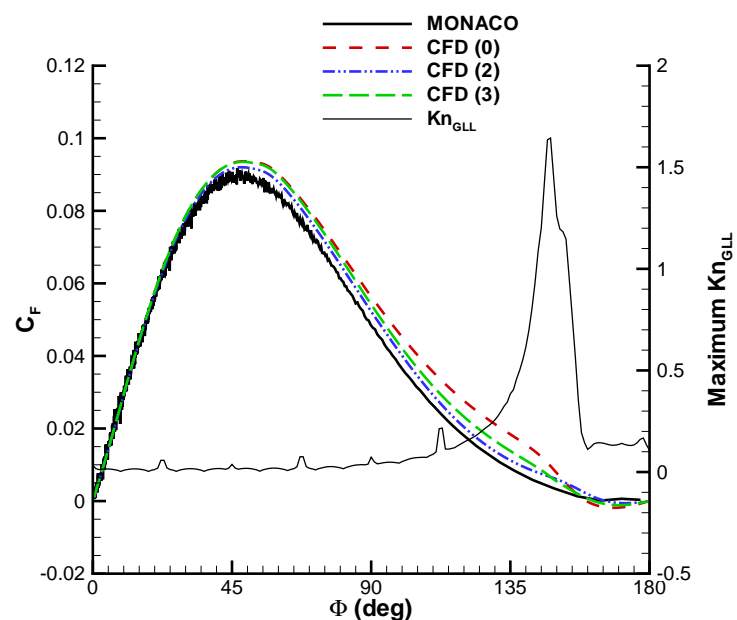

(a) Mach 10

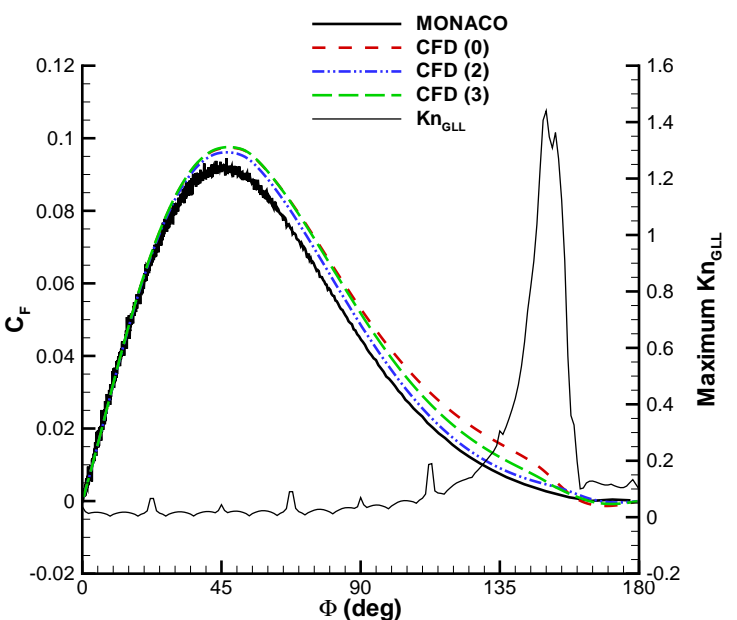

(b) Mach 25

Figure 10. $K n_{\infty}=0.01$ surface friction coefficient and $\mathrm{Kn}_{\mathrm{GLL}}$ for Mach 10 and Mach 25. 


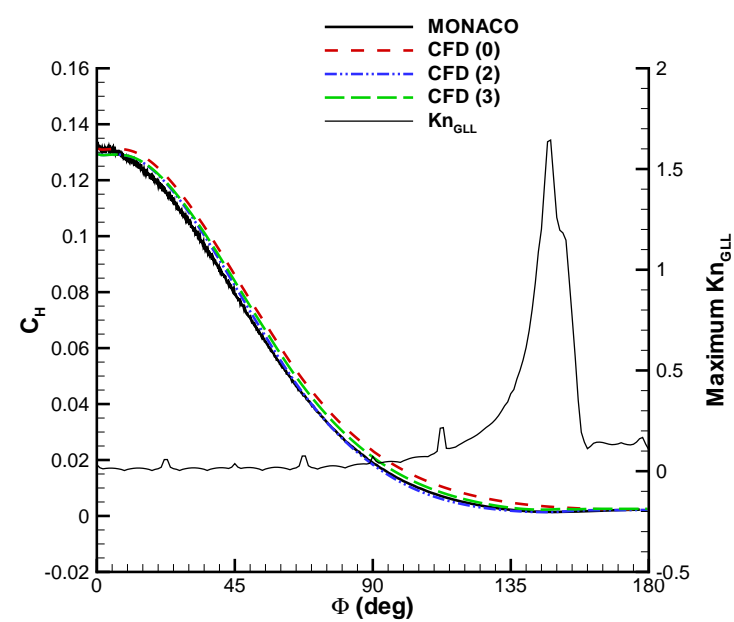

(a) Mach 10

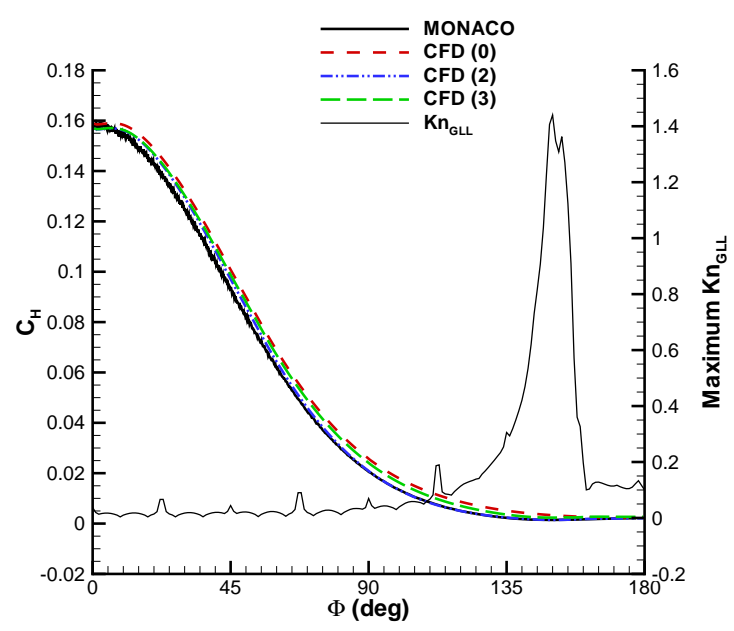

(b) Mach 25

Figure 11. $K \mathrm{n}_{\infty}=0.01$ surface heating coefficient and $\mathrm{Kn}_{\mathrm{GLL}}$ for Mach 10 and Mach 25.

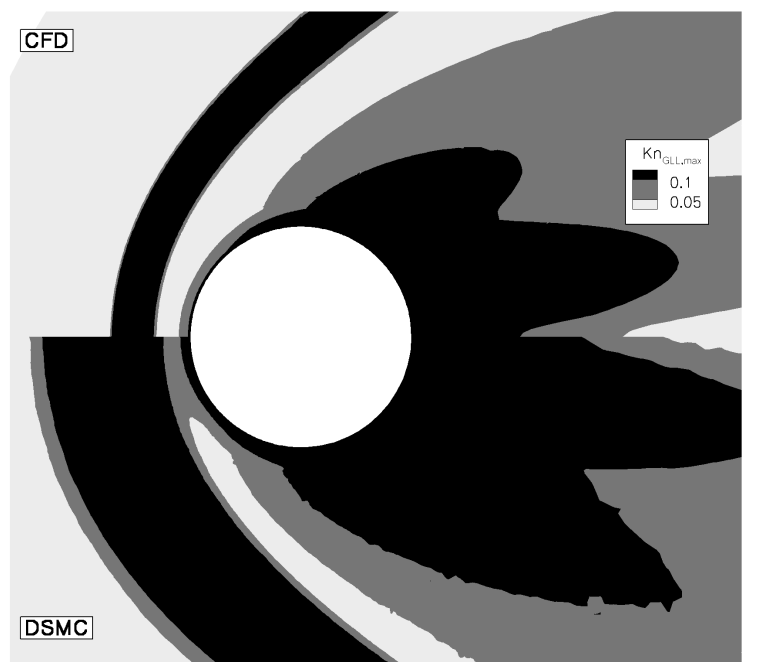

(a) Mach 10

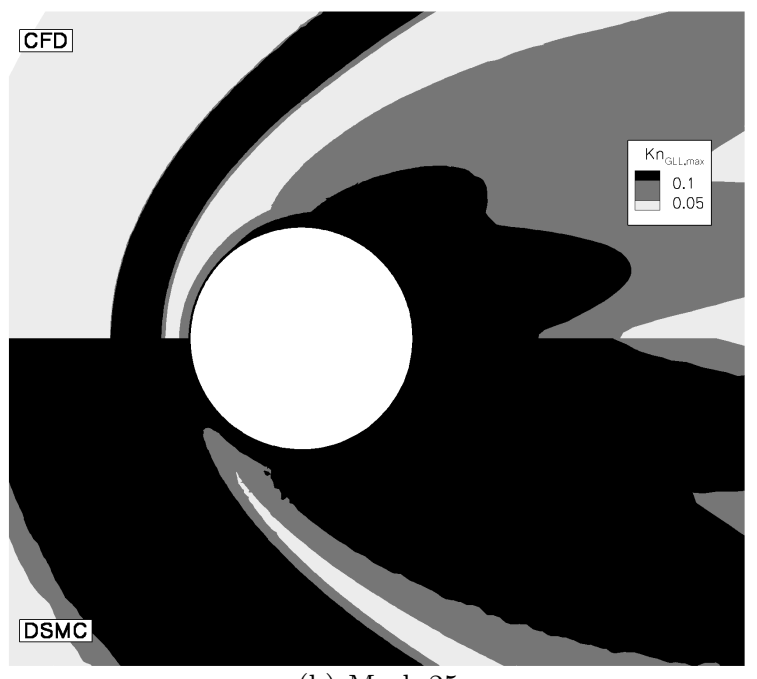

(b) Mach 25

Figure 12. $\mathrm{Kn}_{\infty}=0.05 \mathrm{Kn}_{\mathrm{GLL}}$ for Mach 10 and Mach 25. Light gray regions correspond to $\mathrm{Kn}_{\mathrm{GLL}}<0.05$, dark gray regions correspond to $0.05<\mathrm{Kn}_{\mathrm{GLL}}<0.10$ and black regions correspond to $\mathrm{Kn}_{\mathrm{GLL}}>0.10$. 


\section{VI. $\quad \mathrm{Kn}=0.05$}

At a Knudsen number of 0.05 , the flow is well within the slip regime. The flow demonstrates breakdown in a larger area of the flow, in each of the three regions (shock, boundary layer and wake), as seen in Figure 12. Again, there are larger areas in the Mach 25 case where the breakdown parameter exceeds the critical value of 0.05 ; in fact, nearly the entire domain is predicted to be beyond the region of applicability of the continuum equations. The stagnation line temperatures shown in Figure 13 show a large amount of non-equilibrium present, with only a small amount of vibrational excitation (for the Mach 10 case, the only excitation of vibrational modes is due to the boundary condition at the wall, where the vibrational temperature is set at $500 \mathrm{~K}$ ). The differences between the CFD and DSMC temperature fields shown in Figure 14 are more pronounced than the lower Knudsen number cases, with even more differences for the higher Mach number case. There are significant differences in the vibrational temperature fields, as well (Figure 15). Here, again, it is evident that the vibrational excitation in the Mach 10 flow is due to the wall boundary condition, while there is a higher level of vibrational excitation in the Mach 25 flow due to the higher temperatures present. The DSMC shock is much thicker than the CFD shock, although the shock stand-off distance and peak temperatures are still very nearly equal.

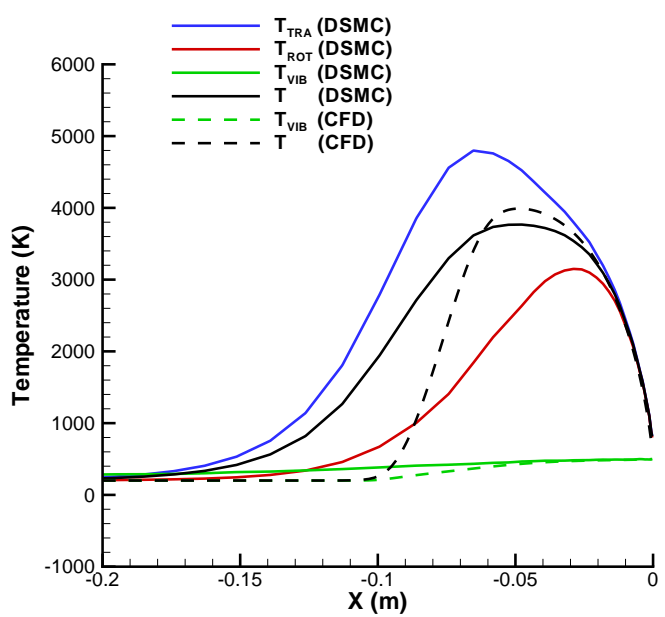

(a) Mach 10

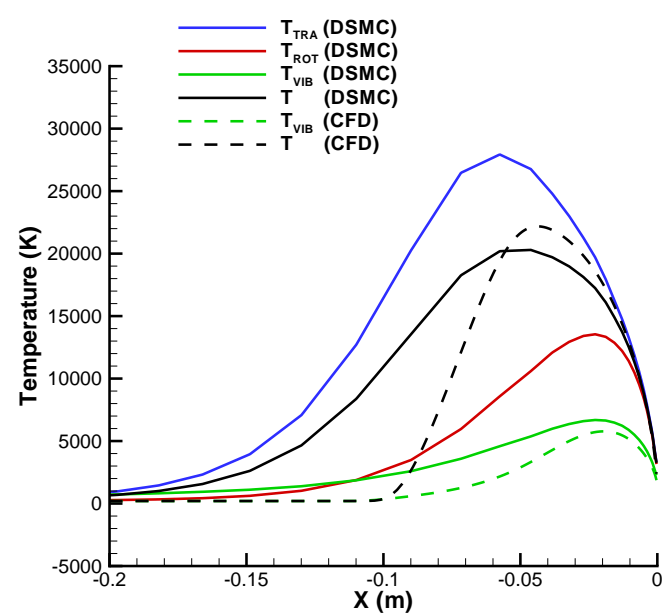

(b) Mach 25

Figure 13. $\mathrm{Kn}_{\infty}=0.05$ temperatures along stagnation line for Mach 10 and Mach 25.

As seen in the previous argon results, the surface pressure predicted by both methods is still in excellent agreement, as seen in Figure 16. However, the shear stress is higher for most of the CFD cases, especially in the wake (see Figure 17). The total drag for all of the slip cases are within $4 \%$ of the DSMC case, with the Type 2 case showing the best agreement. The heat transfer rate, shown in Figure 18, also shows better agreement between the Type 2 case and DSMC. While the no-slip case predicts a peak heat transfer rate about $9 \%$ higher than DSMC, the slip cases show better agreement, with the Type 2 case being within $5 \%$.

The solution temperatures (including the translational, rotational, vibrational and translational/rotational averaged temperature) are plotted along a line normal to the surface at an angle of $90^{\circ}$ as shown in Figures 19 and 20. Although there is some translational-rotational non-equilibrium, there is reasonable agreement between the Type 2 and Type 3 solutions and DSMC, with the Type 3 solutions being slightly better. There is also reasonable agreement between the CFD solutions and the DSMC solution for vibrational temperature, although the CFD method slightly under-predicts the vibrational temperature.

\section{VII. $\quad \mathrm{Kn}=0.25$}

For $\mathrm{Kn}_{\infty}=0.25$, the flow is considered outside of the slip regime and into the transition regime. Here, even the addition of slip boundary conditions does not help the continuum CFD method's predictive capabilities much. Indeed, the plots of the breakdown parameter in Figure 21 indicate that there are significant nonequilibrium effects across almost all of the flow domain. The stagnation line temperature seen in Figure 22 


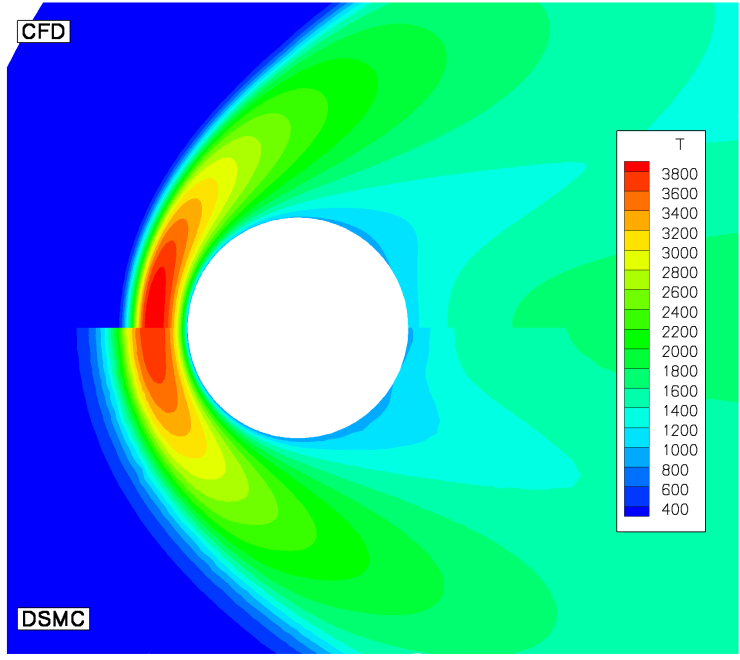

(a) Mach 10

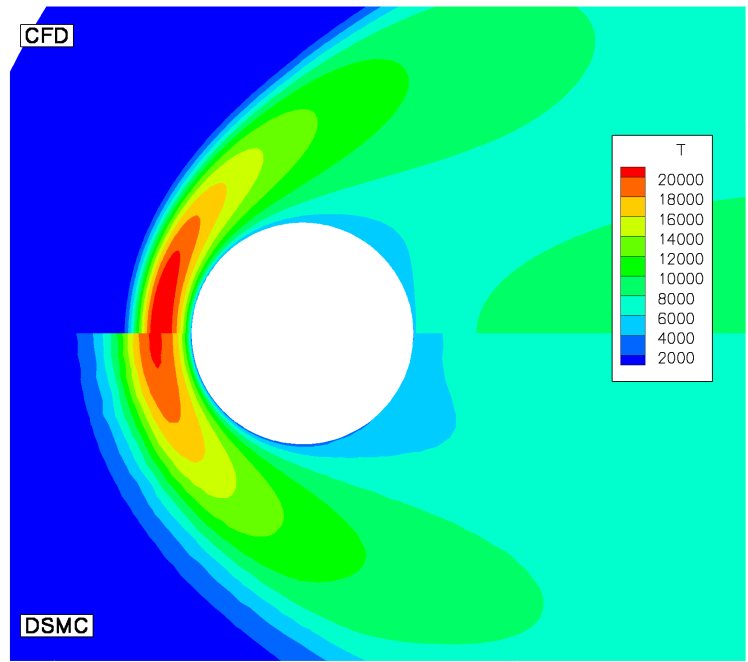

(b) Mach 25

Figure 14. $\mathrm{Kn}_{\infty}=0.05$ translational/rotational temperature field for Mach 10 and Mach 25.

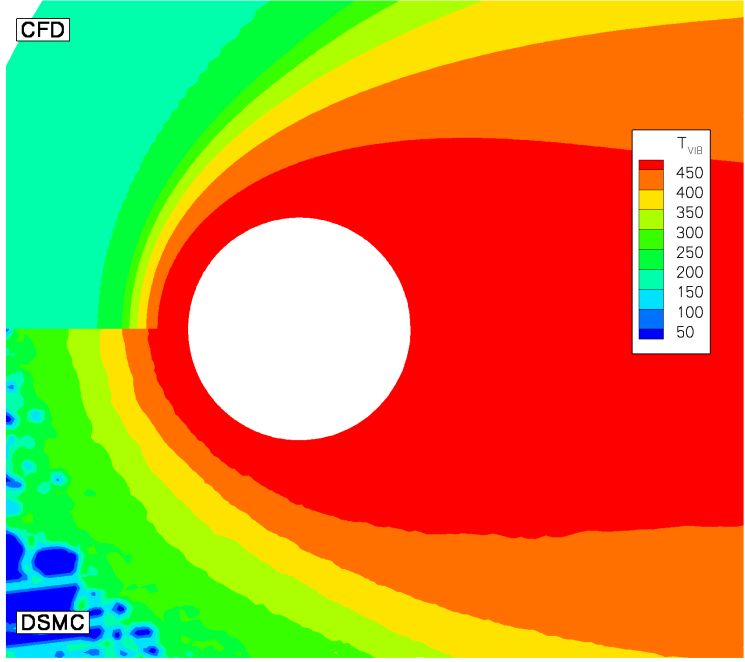

(a) Mach 10

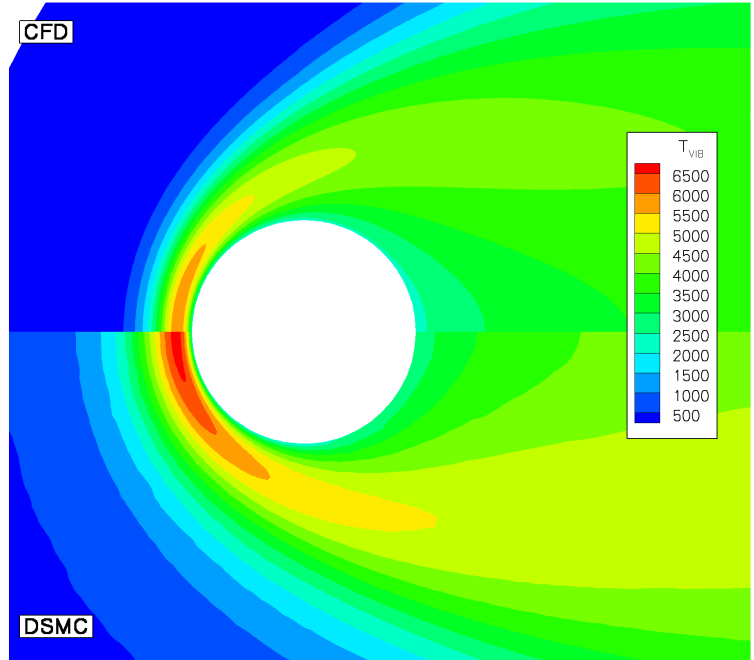

(b) Mach 25

Figure 15. $\mathrm{Kn}_{\infty}=0.05$ vibrational temperature field for Mach 10 and Mach 25. 


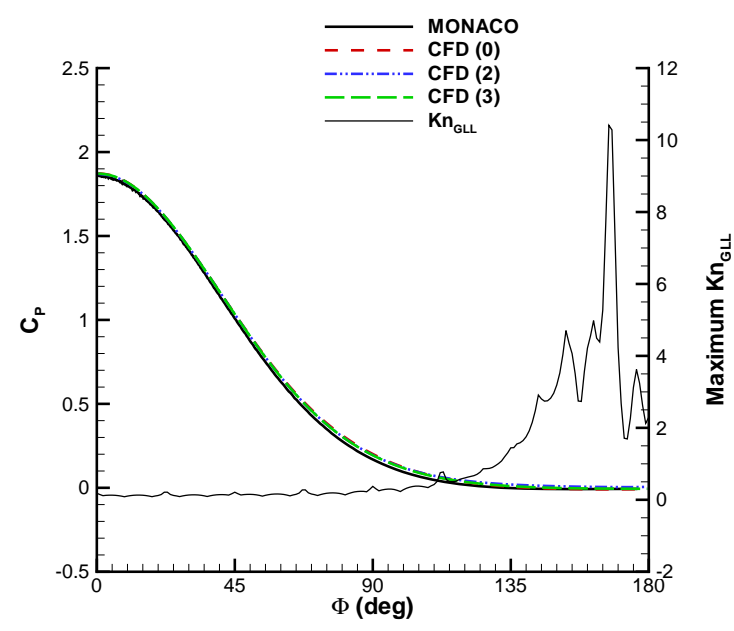

(a) Mach 10

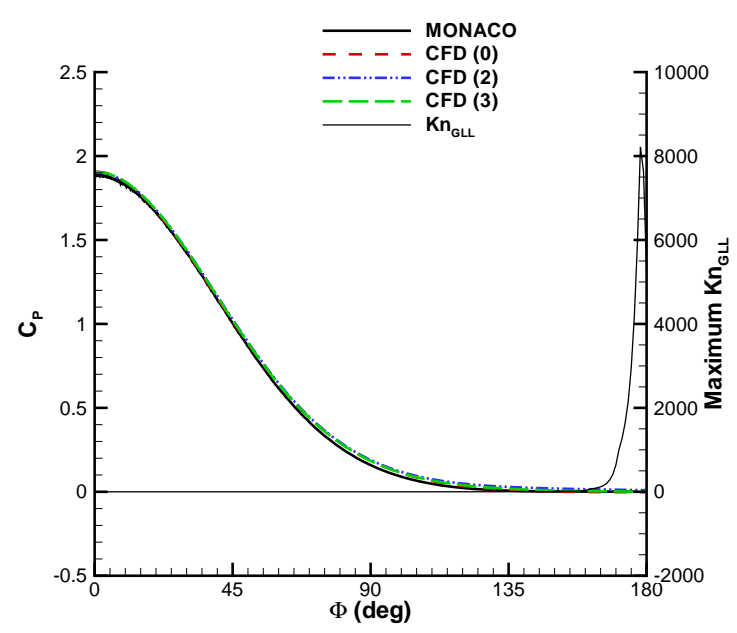

(b) Mach 25

Figure 16. $\mathrm{Kn}_{\infty}=0.05$ surface pressure coefficient and $\mathrm{Kn}_{\mathrm{GLL}}$ for Mach 10 and Mach 25 .

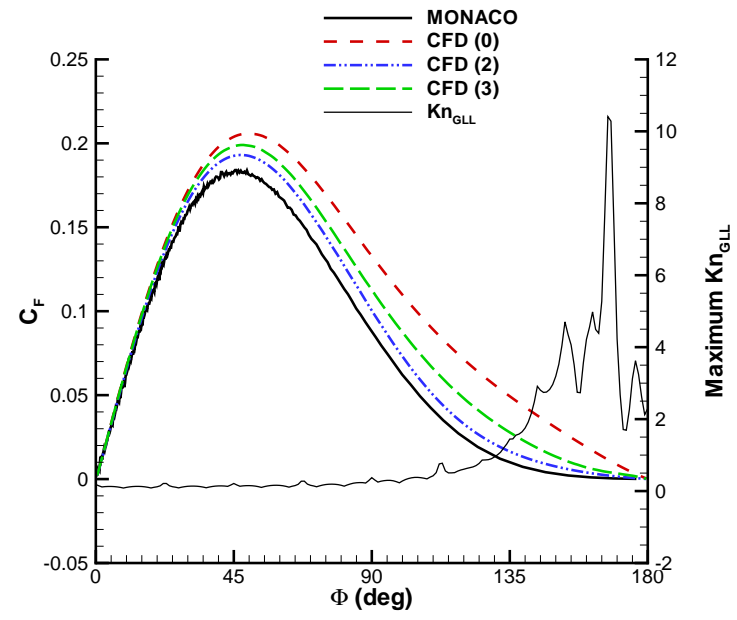

(a) Mach 10

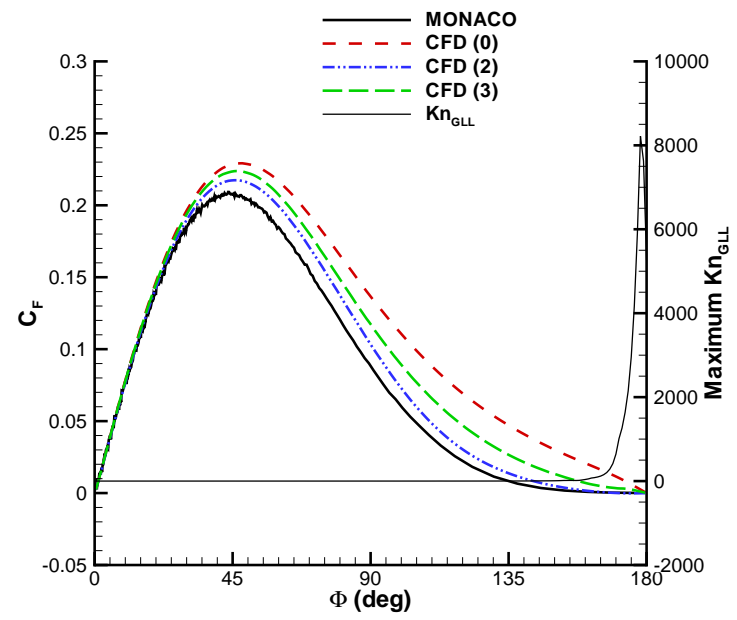

(b) Mach 25

Figure 17. $\mathrm{Kn}_{\infty}=0.05$ surface friction coefficient and $\mathrm{Kn}_{\mathrm{GLL}}$ for Mach 10 and Mach 25. 


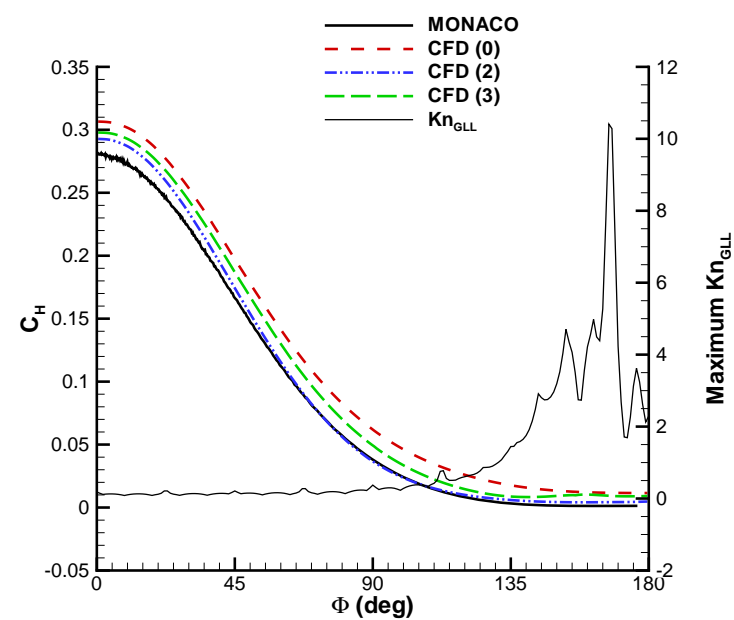

(a) Mach 10

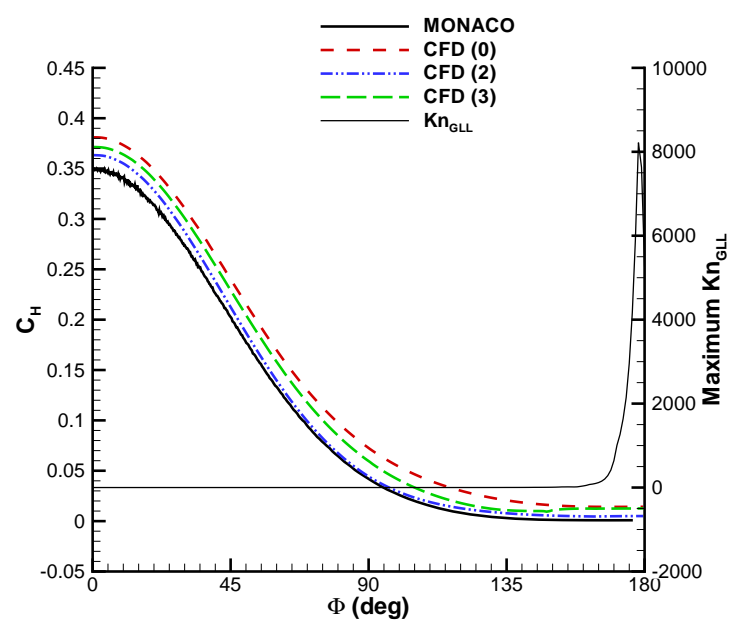

(b) Mach 25

Figure 18. $K \mathbf{n}_{\infty}=0.05$ surface heating coefficient and $K n_{G L L}$ for Mach 10 and Mach 25.

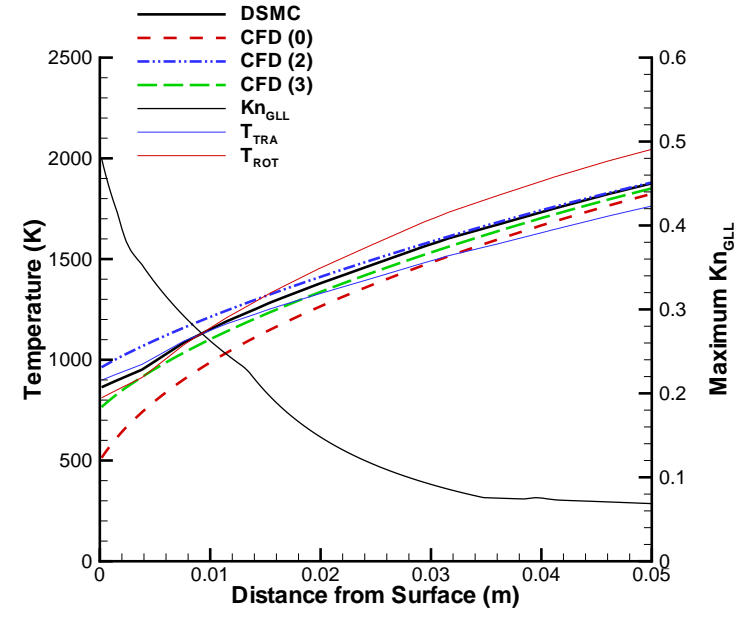

(a) Mach 10

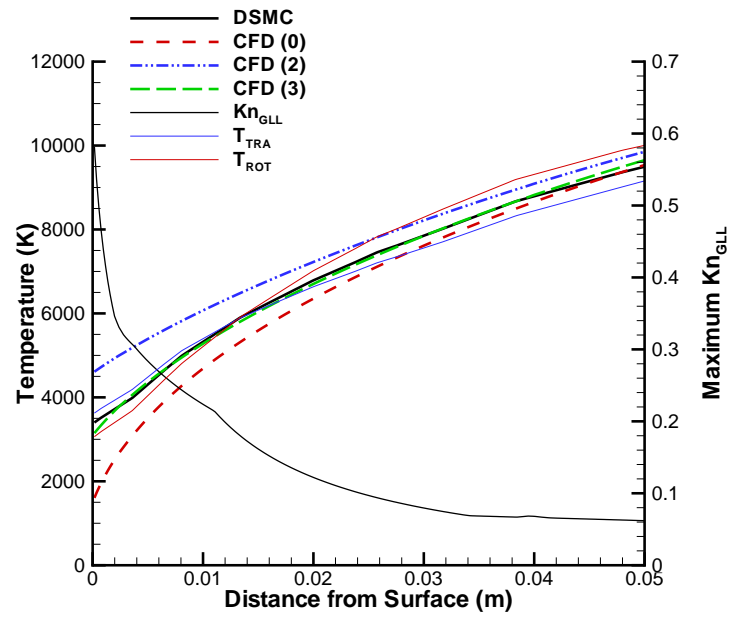

(b) Mach 25

Figure 19. $\mathrm{Kn}_{\infty}=0.05$ translational/rotational temperature and $\mathrm{Kn}_{\mathrm{GLL}}$ along a line normal to body surface at $\Phi=90^{\circ}$. 


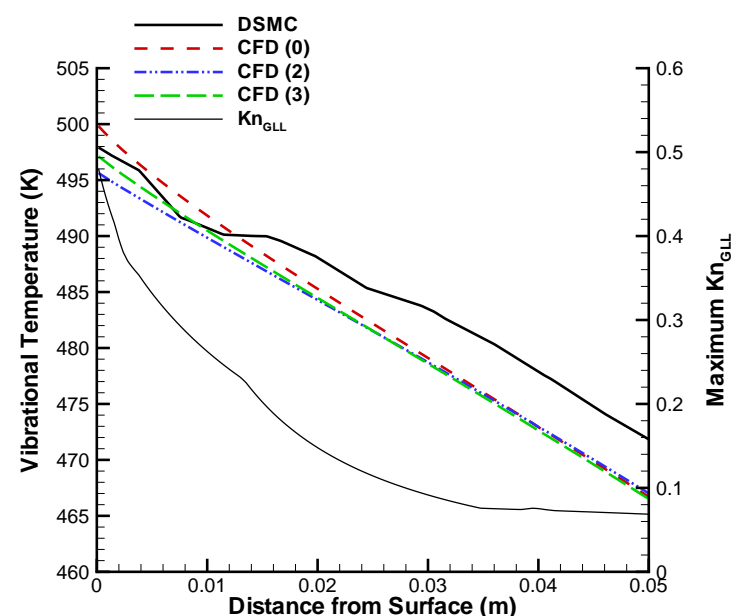

(a) Mach 10

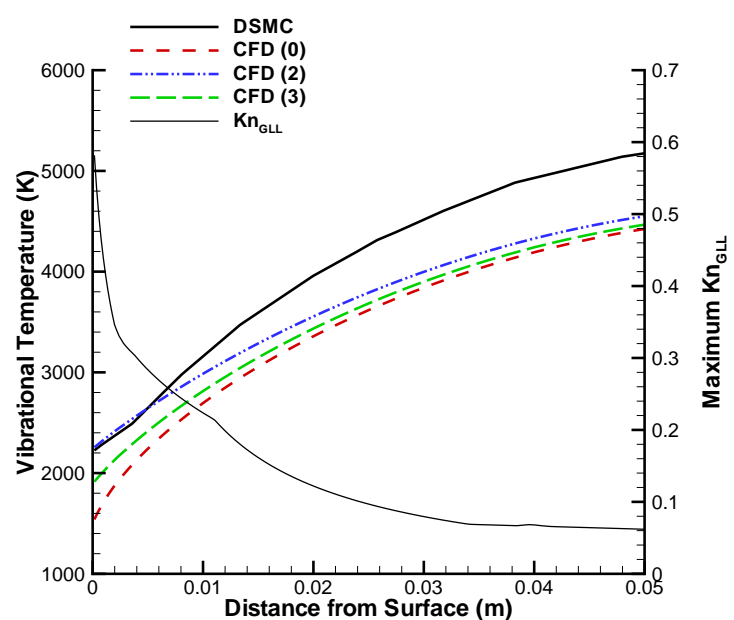

(b) Mach 25

Figure 20. $\mathrm{Kn}_{\infty}=\mathbf{0 . 0 5}$ vibrational temperature and $\mathrm{Kn}_{\mathrm{GLL}}$ along a line normal to body surface at $\Phi=90^{\circ}$.

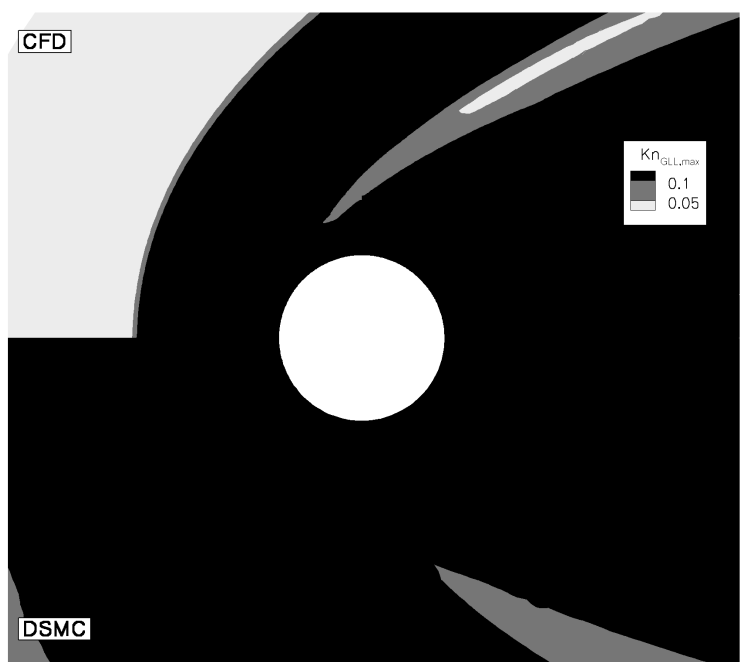

(a) Mach 10

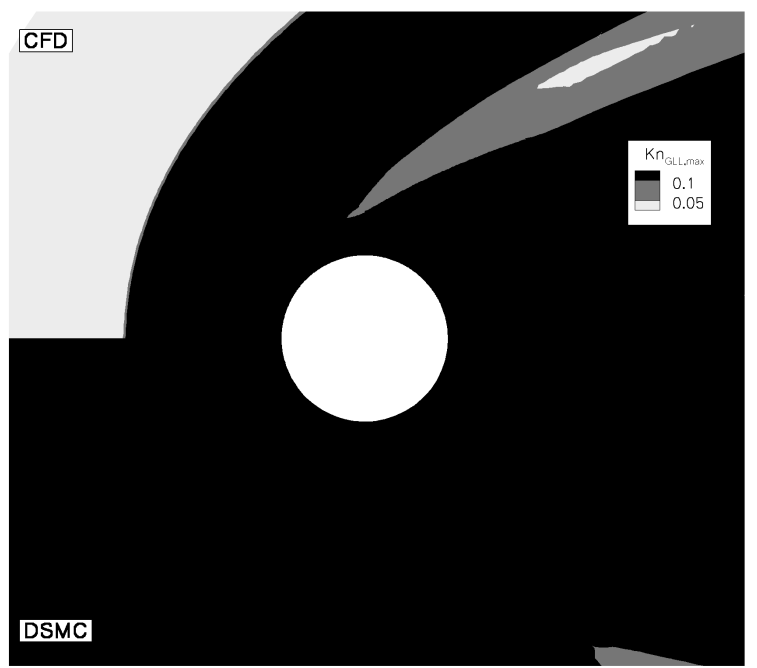

(b) Mach 25

Figure 21. $\mathrm{Kn}=0.25 \mathrm{Kn}_{\mathrm{GLL}}$ for Mach 10 and Mach 25. Light gray regions correspond to $\mathrm{Kn}_{\mathrm{GLL}}<0.05$, dark gray regions correspond to $0.05<\mathrm{Kn}_{\mathrm{GLL}}<0.10$ and black regions correspond to $\mathrm{Kn}_{\mathrm{GLL}}>0.10$. 
shows that the translational-rotational non-equilibrium in the shock extends to the stagnation point. There is also very little vibrational excitation. The temperature fields predicted by both methods, seen in Figure 23, show some major differences. Although the peak temperatures behind the shock appear to be about the same, the shock stand-off distances are significantly different. The vibrational temperature fields shown in Figure 24 show that the CFD solution under-predicts the vibrational temperature.

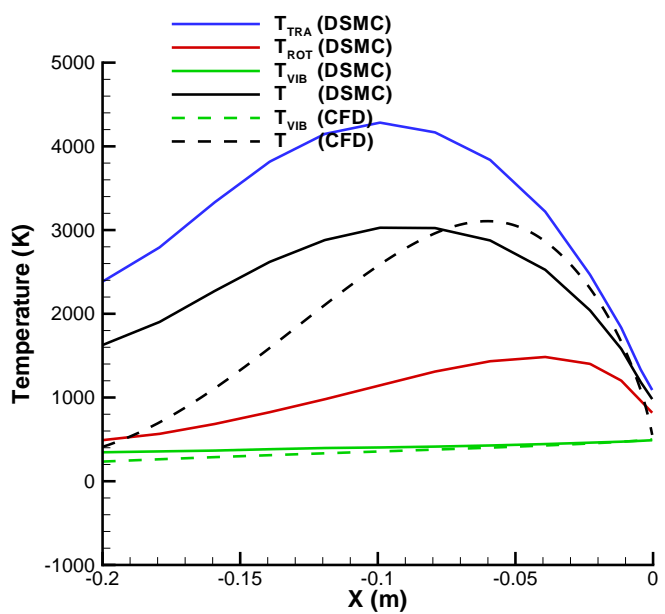

(a) Mach 10

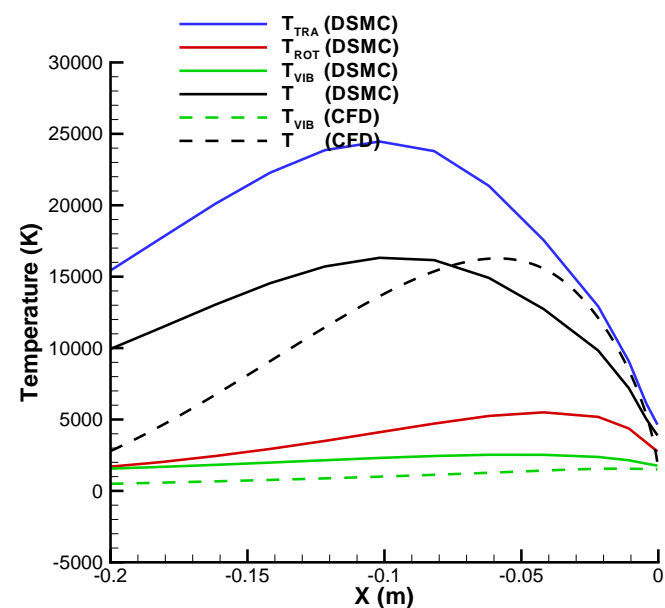

(b) Mach 25

Figure 22. $\mathrm{Kn}=0.25$ temperatures along stagnation line for Mach 10 and Mach 25.

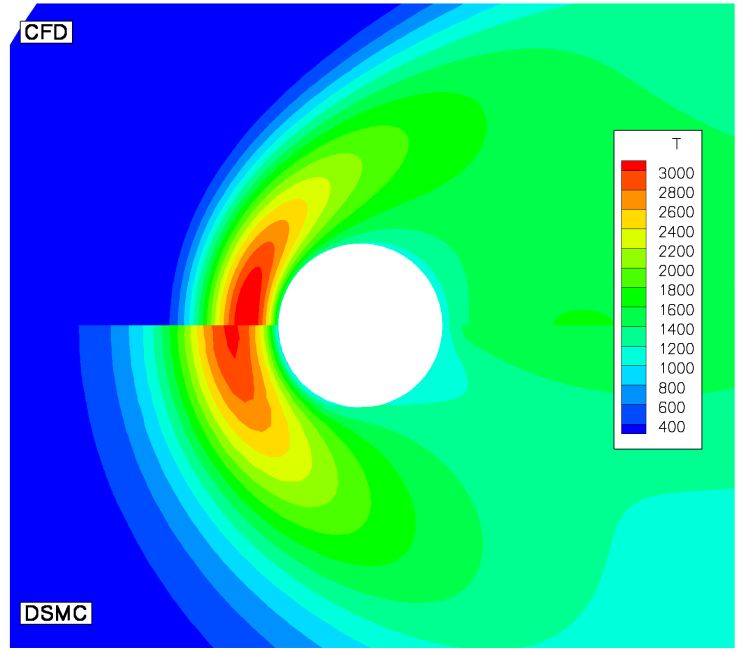

(a) Mach 10

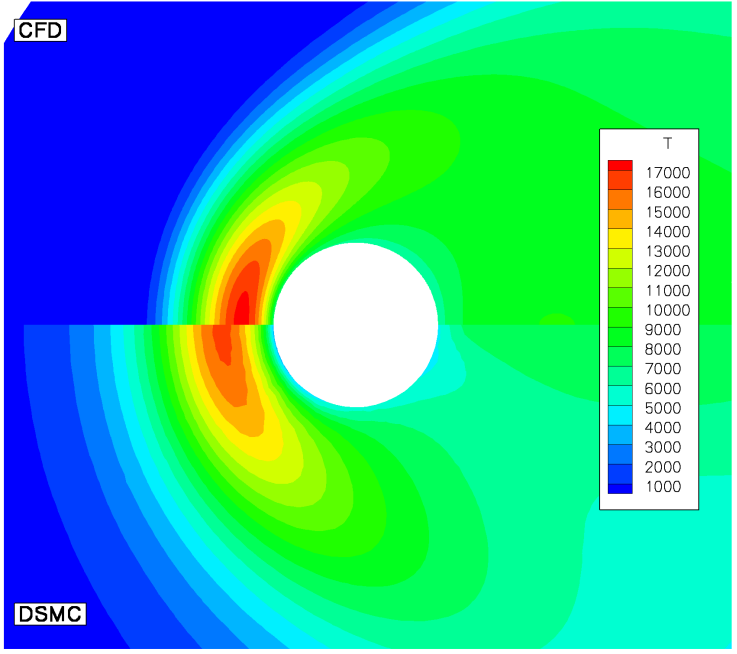

(b) Mach 25

Figure 23. $\mathrm{Kn}=0.25$ translational/rotational temperature field for Mach 10 and Mach 25 .

The surface pressure, plotted in Figure 25, predicted by both methods is no longer in agreement. As with the previous argon analysis the DSMC pressure is less than the CFD pressure near the fore-body (most likely due to the thinner shock predicted by CFD, which allows the CFD solution to approach the ideal jump condition more closely than the DSMC solution). The shear stress, shown in Figure 26, shows the same general trend as in previous cases in that both methods agree near the stagnation region, but the results diverge as the flow accelerates around the cylinder. The heat transfer rate, as seen in Figure 27, follows trends similar to the previous cases in that the DSMC heat transfer rate is lower than the CFD rate along the entire surface for all but the Type 2 case, which gets fairly close in some areas. The peak heat transfer rates differ by as much as almost 30\% for the no-slip case at Mach 25, but the Type 2 case shows the best agreement with just over 5\% difference at Mach 10 and $8 \%$ difference at Mach 25 . 


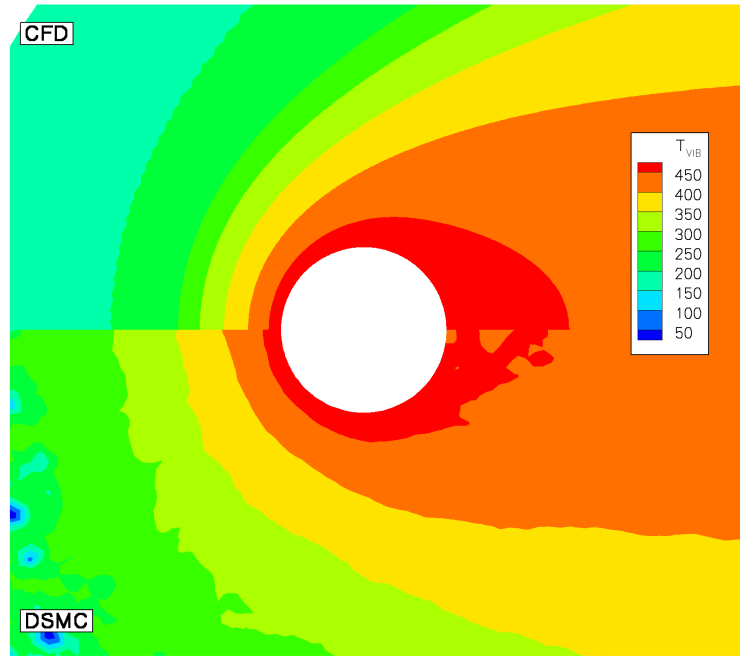

(a) Mach 10

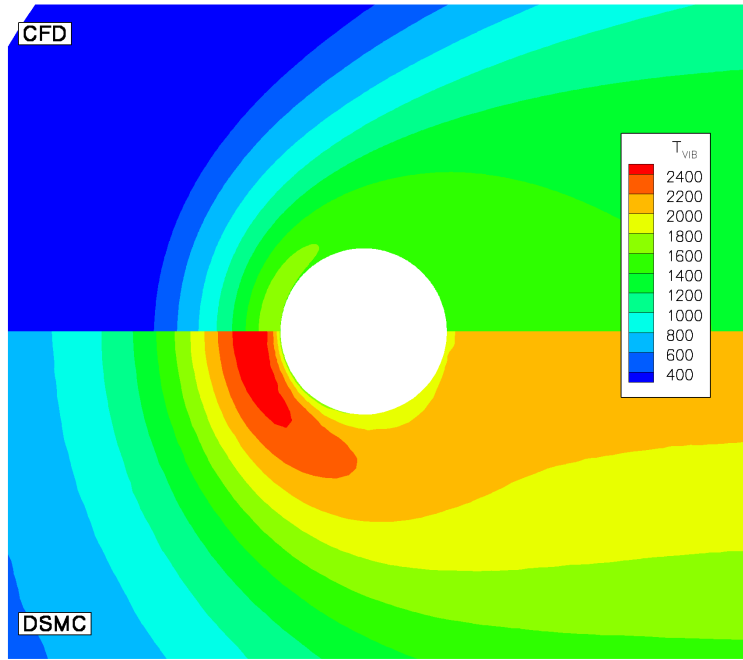

(b) Mach 25

Figure 24. $\mathrm{Kn}=\mathbf{0 . 2 5}$ vibrational temperature field for Mach 10 and Mach 25.

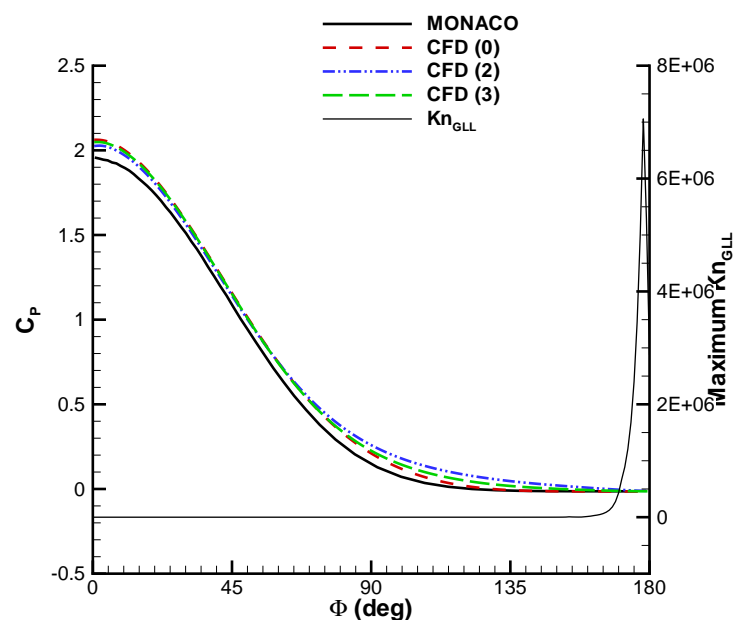

(a) Mach 10

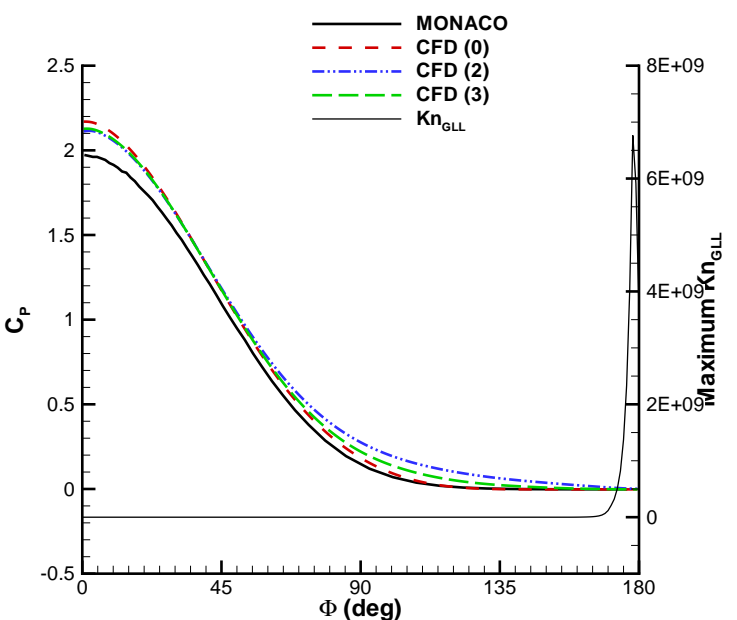

(b) Mach 25

Figure 25. $\mathrm{Kn}_{\infty}=0.25$ surface pressure coefficient and $\mathrm{Kn}_{\mathrm{GLL}}$ for Mach 10 and Mach 25 . 


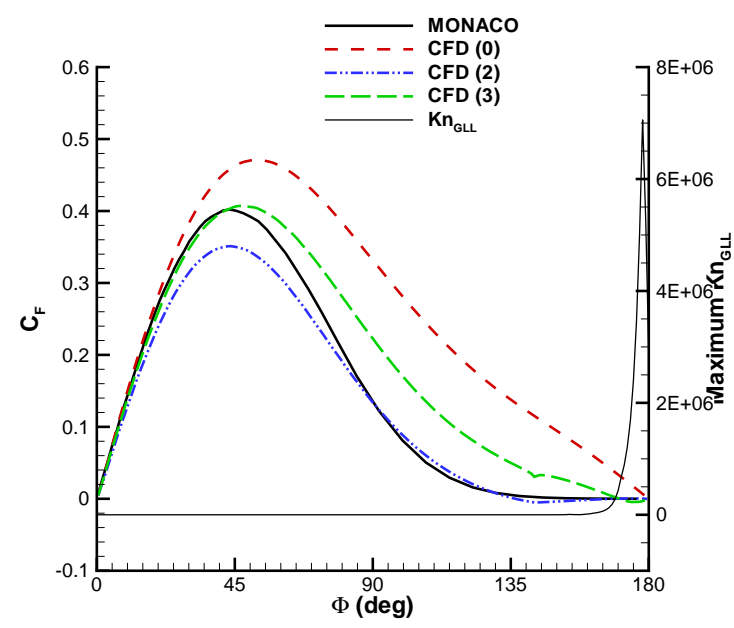

(a) Mach 10

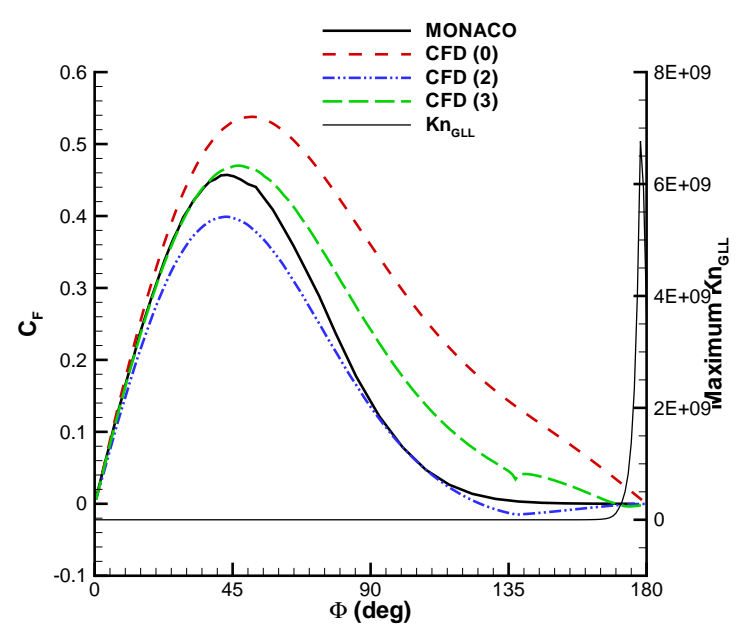

(b) Mach 25

Figure 26. $K n_{\infty}=0.25$ surface friction coefficient and $K n_{G L L}$ for Mach 10 and Mach 25 .

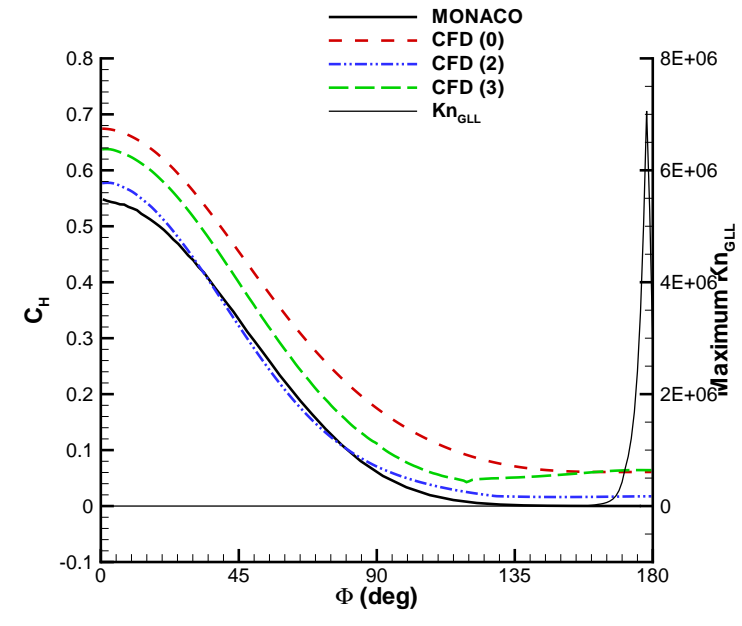

(a) Mach 10

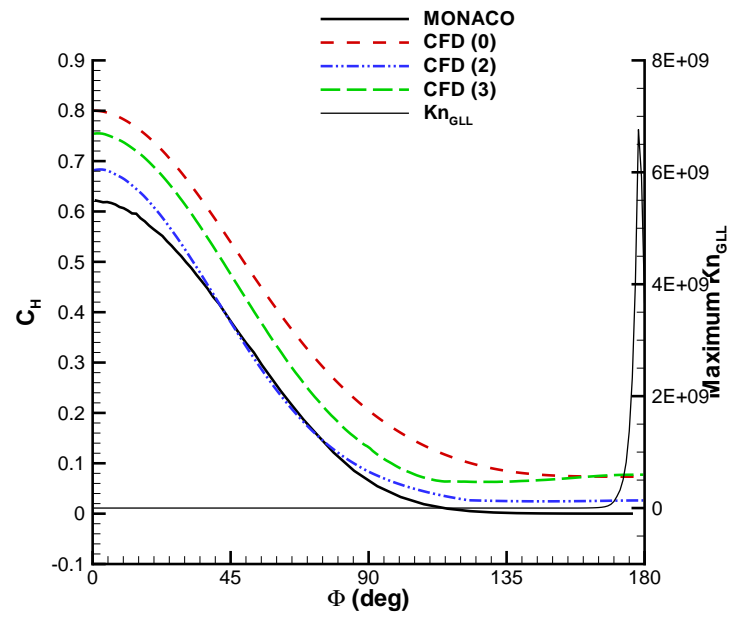

(b) Mach 25

Figure 27. $K n_{\infty}=0.25$ surface heating coefficient and $K_{\mathrm{GLL}}$ for Mach 10 and Mach 25. 


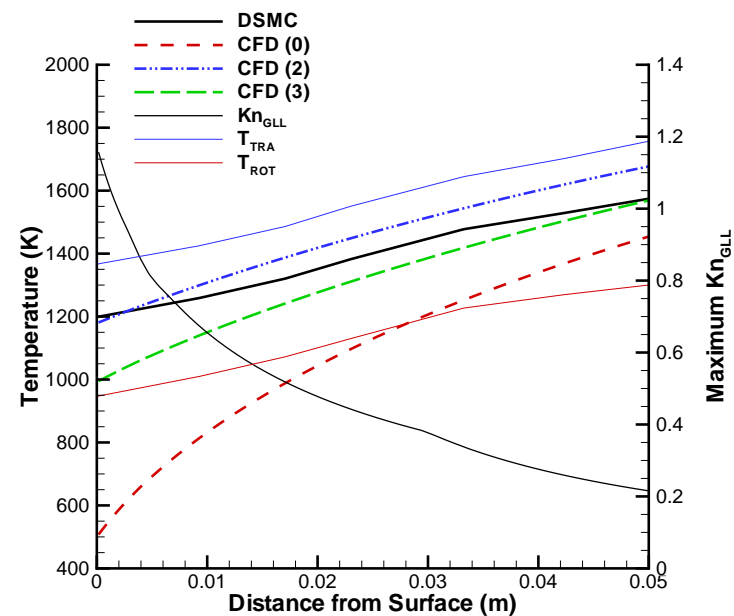

(a) Mach 10

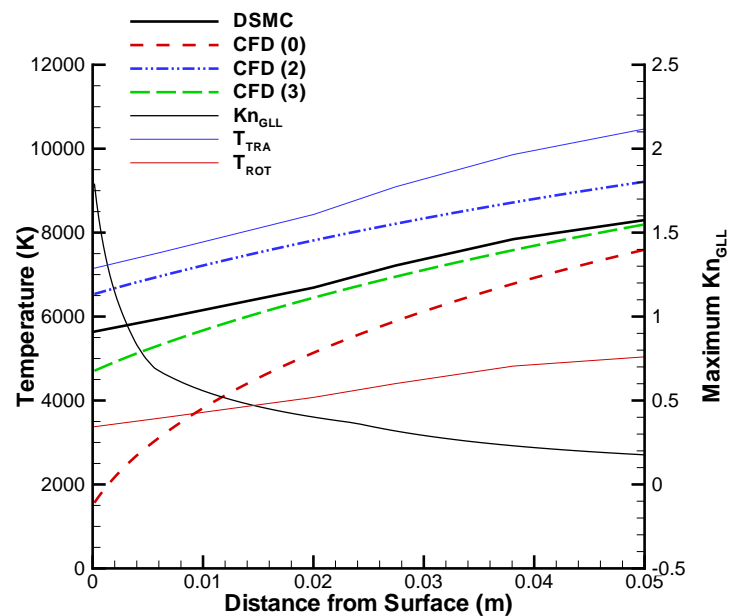

(b) Mach 25

Figure 28. $\mathrm{Kn}_{\infty}=\mathbf{0 . 2 5}$ translational/rotational temperature and $\mathrm{Kn}_{\mathrm{GLL}}$ along a line normal to body surface at $\Phi=90^{\circ}$.

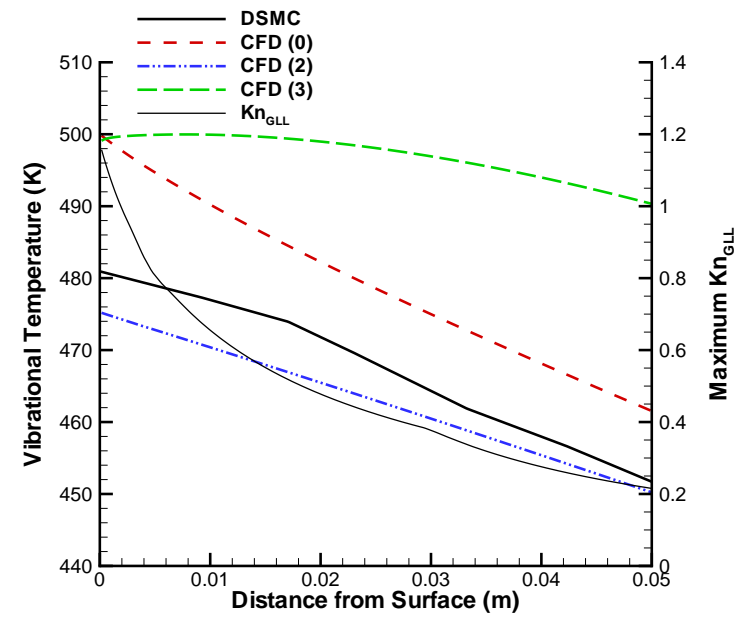

(a) Mach 10

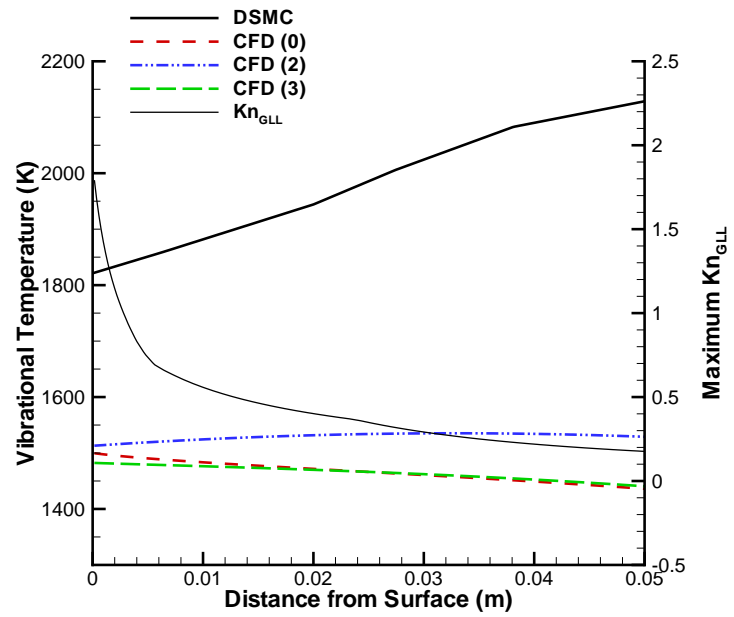

(b) Mach 25

Figure 29. $K \mathrm{~K}_{\infty}=\mathbf{0 . 2 5}$ vibrational temperature and $\mathrm{Kn}_{\mathrm{GLL}}$ along a line normal to body surface at $\Phi=90^{\circ}$. 
The temperatures and breakdown parameter plotted along the line normal to the surface at an angle of $90^{\circ}$ are shown in Figures 28 (translational/rotational) and 29 (vibrational). There is a significant amount of translational-rotational non-equilibrium predicted by DSMC due to the rarefied flow conditions. For translational/rotational temperature results, both the Type 2 and Type 3 boundary conditions show reasonable agreement with DSMC. Figure 29 shows some interesting effects of the vibrational temperature jump boundary condition. Although DSMC predicts a fairly small negative vibrational temperature jump at the wall for the Mach 10 case, the Type 2 boundary condition predicts a larger negative temperature jump and the Type 3 boundary condition predicts a negligibly small temperature jump. However, the se differences are actually small in magnitude; the actual vibrational temperatures predicted by each simulation vary by only about $50 \mathrm{~K}$ along this line. For the Mach 25 case, though, DSMC predicts a positive vibrational temperature jump while the Type 2 boundary condition solution shows a much smaller positive vibrational temperature jump and the Type 3 case predicts a small negative vibrational temperature jump.

\section{Conclusion}

The primary goal of this study was to quantify the difference between DSMC and CFD simulations for a gas with internal energy to determine the effects of different levels of non-equilibrium on surface properties under hypersonic flow conditions.

In addition to ensuring similar treatment of transport quantities (such as viscosity and thermal conductivity), it was also necessary to ensure similar treatment of vibrational relaxation. It was shown that although the MONACO model for translational-vibrational energy exchange seeks to accurately reproduce the Landau-Teller model for vibrational relaxation, it was necessary to multiply the vibrational collision probability by a factor that depends on the maximum translational temperature in the flow field. With the use of this factor, excellent agreement between CFD and DSMC prediction of the vibrational temperature was obtained at the lowest Knudsen number conditions.

Although there was a significant amount of non-equilibrium between the different thermal modes (translational, rotational and vibrational), the trends shown here were largely similar to the trends noted previously when considering a simple gas with no internal degrees of freedom. The pressure and shear stress were least sensitive to the non-equilibrium effects, while the heat transfer rate was most sensitive. For surface properties, the CFD results using the Type 2 boundary conditions showed the best agreement with DSMC, with reasonable agreement for flow properties as well.

\section{Acknowledgments}

A. J. Lofthouse gratefully acknowledges the support of the Air Force Institute of Technology. This work is also sponsored in part by the Space Vehicle Technology Institute, under NASA grant NCC3-989 with joint sponsorship from the Department of Defense, and by the Air Force Office of Scientific Research, through grant FA9550-05-1-0115. The generous use of NASA high performance computing resources was indispensable to this investigation and is greatly appreciated.

\section{References}

${ }^{1}$ Vincenti, W. G. and C. H. Kruger, J., Introduction to Physical Gas Dynamics, Krieger Publishing Company, 1965.

${ }^{2}$ Bird, G. A., Gas Dynamics and the Direct Simulation of Gas Flows, Oxford University Press, Oxford, 1994.

${ }^{3}$ Wagner, W., "A convergence proof for Bird's direct simulation Monte Carlo method for the Boltzmann equation," Journal of Statistical Physics, Vol. 66, No. 3-4, Feb. 1992, pp. 1011-1044.

${ }^{4}$ Bird, G. A., "Breakdown of Translational and Rotational Equilibrium in Gaseous Expansions," AIAA Journal, Vol. 8, No. 11, 1970, pp. 1998-2003.

${ }^{5}$ Tiwari, S., "Coupling of the Boltzmann and Euler Equations with Automatic Domain Decomposition," Journal of Computational Physics, Vol. 144, Aug. 1998, pp. 710-726.

${ }^{6}$ Garcia, A. L., Bell, J. B., Crutchfield, W. Y., and Alder, B. J., "Adaptive Mesh and Algorithm Refinement Using Direct Simulation Monte Carlo," Journal of Computational Physics, Vol. 154, 1999, pp. 134.

${ }^{7}$ Camberos, J. A., Schrock, C. R., McMullan, R. J., and Branam, R. D., "Development of Continuum Onset Criteria with Direct Simulation Monte-Carlo Using Boltzmann's H-Theorem: Review and Vision," Proceedings of the 9th AIAA/ASME Joint Thermophysics and Heat Transfer Conference, San Francisco, California, June 2006.

${ }^{8}$ Boyd, I. D., Chen, G., and Candler, G. V., "Predicting Failure of the Continuum Fluid Equations in Transitional Hypersonic Flows," Physics of Fluids, Vol. 7, No. 1, Jan. 1995, pp. 210-219. 
${ }^{9}$ Wang, W.-L., A Hybrid Particle/Continuum Approach for Nonequilibrium Hypersonic Flows, Phd thesis, The University of Michigan, 2004

${ }^{10}$ Lofthouse, A. J., Scalabrin, L. C., and Boyd, I. D., "Velocity Slip and Temperature Jump in Hypersonic Aerothermodynamics," AIAA Paper 2007-0208, presented at the 45th AIAA Aerospace Sciences Meeting and Exhibit, Reno, NV, Jan. 2007.

${ }^{11}$ Lofthouse, A. J., Boyd, I. D., and Wright, M. J., "Effects of continuum breakdown on hypersonic aerothermodynamics," Physics of Fluids, Vol. 19, No. 2, 2007, pp. 027105.

${ }^{12}$ Dietrich, S. and Boyd, I. D., "Scalar and Parallel Optimized Implementation of the Direct Simulation Monte Carlo Method," Journal of Computational Physics, Vol. 126, No. 2, 1996, pp. 328-342.

${ }^{13}$ Koura, K. and Matsumoto, H., "Variable Soft Sphere Molecular Model for Air Species," Physics of Fluids A, Vol. 4, No. 5, May 1992, pp. 1083-1085.

${ }^{14}$ Vijayakumar, P., Sun, Q., and Boyd, I. D., "Vibrational-Translational Energy Exchange for the Direct Simulation Monte Carlo Method," Physics of Fluids, Vol. 11, No. 8, Aug. 1999, pp. 2117-2126.

${ }^{15}$ Boyd, I. D., "Analysis of Rotational Nonequilibrium in Standing Shock Waves of Nitrogen," AIAA Journal, Vol. 28, No. 11, Nov. 1990, pp. 1997-1999.

${ }^{16}$ Scalabrin, L. C. and Boyd, I. D., "Development of an Unstructured Navier-Stokes Solver for Hypersonic Nonequilibrium Aerothermodynamics," AIAA Paper 2005-5203, presented at the 38th AIAA Thermophysics Conference, Toronto, Ontario Canada, June 2005.

${ }^{17}$ Scalabrin, L. C. and Boyd, I. D., "Numerical Simulation of Weakly Ionized Hypersonic Flow for Reentry Configurations," AIAA Paper 2006-3773, presented at the 9th AIAA/ASME Joint Thermophysics and Heat Transfer Conference, San Fransisco, CA, June 2006.

${ }^{18}$ Park, C., Nonequilibrium Hypersonic Aerothermodynamics, John Wiley \& Sons, 1990.

${ }^{19}$ Wilke, C. R., "A Viscosity Equation for Gas Mixtures," Journal of Chemical Physics, Vol. 18, No. 4, April 1950, pp. 517-519.

${ }^{20}$ Blottner, F. G., Johnson, M., and Ellis, M., "Chemically Reacting Viscous Flow Program for Multi-Component Gas Mixtures," Tech. Rep. SC-RR-70-754, Sandia Laboratories, Albuquerque, New Mexico, 1971.

${ }^{21}$ Schwartzentruber, T. E., Scalabrin, L. C., and Boyd, I. D., "Hybrid Particle-Continuum Simulations of Non-Equilibrium Hypersonic Blunt Body Flow Fields," AIAA Paper 2006-3602, presented at the 9th AIAA/ASME Joint Thermophysics and Heat Transfer Conference, San Francisco, CA, June 2006.

${ }^{22}$ Gökçen, T. and MacCormack, R. W., "Nonequilibrium Effects for Hypersonic Transitional Flows Using Continuum Approach," AIAA Paper 1989-0461, presented at the 27th Aerospace Sciences Meeting, Reno, NV, Jan. 1989.

${ }^{23}$ Lockerby, D. A., Reese, J. M., and Gallis, M. A., "Capturing the Knudsen Layer in Continuum-Fluid Models of Nonequilibrium Gas Flows," AIAA Journal, Vol. 43, No. 6, June 2005, pp. 1391-1393.

${ }^{24}$ Gökçen, T., MacCormack, R. W., and Chapman, D. R., "Computational Fluid Dyanmics Near the Continuum Limit," AIAA Paper 1987-1115, 1987.

${ }^{25}$ Millikan, R. C. and White, D. R., "Systematics of Vibrational Relaxation," Journal of Chemical Physics, Vol. 39, 1963, pp. 2309-3213

${ }^{26}$ Boyd, I. D., "Analysis of Vibrational-Translational Energy Transfer Using the Direct Simulation Monte Carlo Method," Physics of Fluids A, Vol. 3, No. 7, July 1991, pp. 1785-1791.

${ }^{27}$ Lumpkin, F. E., Haas, B. L., and Boyd, I. D., "Resolution of Differences Between Collision Number Definitions in Particle and Continuum Simulations," Physics of Fluids A, Vol. 3, No. 9, Sept. 1991, pp. 2282-2284.

${ }^{28}$ Abe, T., "Direct Simulation Monte Carlo Method for Internal-Translational Energy Exchange in Nonequilibrium Flow," Rarefied Gas Dynamics: Theory and Simulations, edited by B. D. Shizgal and D. P. Weaver, Vol. 159 of Progress in Astronautics and Aeronautics, AIAA, 1994, pp. 103-113.

${ }^{29}$ Farbar, E., "Testing of Vibrational Relaxation Model in MONACO," Unpublished report, Department of Aerospace Engineering, University of Michigan. 